LPT-ORSAY 00/31

\title{
Lectures on differentials, generalized differentials and on some examples related to theoretical physics
}

\author{
Michel Dubois-Violette
}

\begin{abstract}
These notes contain a survey of some aspects of the theory of differential modules and complexes as well as of their generalization, that is, the theory of $N$-differential modules and $N$-complexes. Several applications and examples coming from physics are discussed. The commun feature of these physical applications is that they deal with the theory of constrained or gauge systems. In particular different aspects of the BRS methods are explained and a detailed account of the $N$-complexes arising in the theory of higher spin gauge fields is given.
\end{abstract}

\section{Introduction}

Differential algebraic and (co)homological methods have rapidly sprung up in theoretical physics in connection with the development of gauge theories. Their interventions occur at two levels, firstly at a classical level under a more systematic use of the calculus of differential forms, secondly under the emergence of the BRS methods in connection with the quantization of gauge theories. In fact the BRS technique provides an explicitely local and relativistic invariant way to develop perturbation theory for quantum gauge theories [2], 3]. It is worth noticing here that one cannot overestimate the role of the locality principle in perturbative renormalization 24. Independently of these perturbative developments, methods for quantizing constrained systems on phase space have been developed using the path integral 28] which were obviously related. In both cases enter "ghosts" 25 and the occurrence of a differential, i.e. an endomorphism of square zero. It turns out that the latter construction essentially reduces to a "homological" description of classical constrained systems 37 in which the ghosts and the differential have a natural interpretation in terms of standard mathematical concepts [48], [52], [56], [12.

Here we shall not give a systematic exposition of the above topics but, instead, we shall follow a sort of transversal way. These notes give a survey of appropriate concepts and results in homology which will be illustrated at each level with examples of application in theoretical physics. Furthermore recent developments in a generalization of homology will be reviewed as well as some physical applications.

The plan is the following. In Section 2 we give the basic definitions and results on homology of differential modules. In Section 3 we introduce graduation, that is

To be published in the Proceedings of Bariloche 2000, "Quantum Symmetries in Theoretical Physics and Mathematics", R. Coquereaux, R. Trinchero Eds, Contemporary Mathematics, American Mathematical Society. 
we discuss complexes and give several examples; in this section we explain the constructions connected with simplicial modules and we describe the tensor product of complexes. Section 4 is a physical illustration of the fact that there is no natural tensor product of differential modules whereas there is one for complexes; we show there that the introduction of ghosts at the one-particle level in the free field theory is worthwhile to render the theory natural over the physical space. In Section 5 we introduce $N$-differentials and discuss the generalization of homology associated with $N$-differential modules; we give there several examples of constructions some of which are related to physics (e.g. parafermions). Section 6 is devoted to the corresponding graded situation i.e. to $N$-complexes; we recall there the constructions of $N$-complexes associated to simplicial modules and the result which expresses in these cases the generalized homology in terms of the ordinary one (Theorem 2) [14. In Section 7 which summarizes results of [17, [18, we introduce $N$-complexes of tensor fields on $\mathbb{R}^{D}$ generalizing the complex of differential forms and we state the corresponding generalization of the Poincaré lemma (Theorem 3); we also explain why these $N$-complexes naturally enter the theory of higher spin gauge fields. In Section 8 we discuss graded differential algebras and their " $N$-generalization" and give a universal $N$-construction generalizing the usual universal differential calculus over a unital associative algebra [20], 14. Section 9 describes the homological approach to "subquotients" and applies it to constrained systems (BRS-method). The main result, Theorem 4, is slightly more general than the results of [12] (more general context), so we give a sketch of proof of Lemma 10 on which it relies. Finally in Section 10 we generalize constructions of the previous section to $N$-differential modules in connection with a quantum gauge group problem arising for the zero modes in the Wess-Zumino-Novikov-Witten model; this section is a summary of 23] (see also [22]).

These notes contain almost no proof, many results are classical or easy. There are two notable exceptions, namely Theorem 2 and Theorem 3 the proof of which are absolutely non trivial although their meaning is transparent.

Let us say some words on our conventions. For sake of completeness we have given the formulation in terms of modules over a commutative ring $\mathbf{k}$; the tensor product symbol $\otimes$ without other specification means the tensor product over $\mathbf{k}$ (of $\mathbf{k}$-modules), i.e. $\otimes=\otimes_{\mathbf{k}}$. In the physical examples $\mathbf{k}$ is either the field $\mathbb{R}$ of the field $\mathbb{C}$, so the reader may well understand $\mathbf{k}$ like that and then the $\mathbf{k}$-modules are vector spaces over $\mathbb{R}$ or $\mathbb{C}$. We use throughout the Einstein convention of summation of repeated up-down indices. A diagram of mappings between sets is said to be a commutative diagram if given two path of mappings between (two vertex) two sets of the diagram, the corresponding compositions of mappings coincide. A Young diagram is a finite collection of boxes, or cells, arranged in left-justified rows, with a weakly decreasing number of cells in each row. Given a Young diagram of $n$ cells $Y$, one associates to it a projector $\mathbf{Y}$, the Young symmetrizer, on the space of covariant tensors of degree $n$ on $\mathbb{R}^{D}$ by the following procedure. Let $T_{\mu_{1} \cdots \mu_{n}}$ be the components of $T$, then the components $\mathbf{Y}(T)_{\mu_{1} \cdots \mu_{n}}$ of $\mathbf{Y}(T)$ are obtained by filling successively the cells of the rows of $Y$ with $\mu_{1}, \cdots, \mu_{n}$, then by symmetrizing the $\mu$ 's which belong to the same rows and then by antisymmetrizing the $\mu$ 's which belong to the same columns. For Young diagrams etc., we use the notations of 30 . 
We also mention that many subjects of these lectures are also treated in [16] so, although the aims of [16] are different, it is a complement for the present notes.

\section{Differential modules}

Throughout these notes, $\mathbf{k}$ is a commutative ring with a unit and by a module without other specification, we always mean a k-module; the same convention is adopted for homomorphisms, endomorphisms, etc.. A module $E$ equipped with an endomorphism $d$ satisfying $d^{2}=0$ will be referred to as a differential module and the endomorphism $d$ as its differential. Given two differential modules $(E, d)$ and $\left(E^{\prime}, d^{\prime}\right)$, a homomorphism of differential modules of $E$ into $E^{\prime}$ is a homomorphism (of k-modules) $\varphi: E \rightarrow E^{\prime}$ satisfying $\varphi \circ d=d^{\prime} \circ \varphi$.

A sequence of homomorphisms of modules (resp. of differential modules)

$$
\cdots \longrightarrow E_{i} \stackrel{\varphi_{i}}{\longrightarrow} E_{i+1} \stackrel{\varphi_{i+1}}{\longrightarrow} E_{i+2} \longrightarrow \cdots
$$

is said to be exact if $\operatorname{Im}\left(\varphi_{i}\right)=\operatorname{Ker}\left(\varphi_{i+1}\right)$. In particular the sequence $0 \rightarrow E \stackrel{\varphi}{\rightarrow} F$ is exact if and only if $\varphi$ is injective and the sequence $E \stackrel{\varphi}{\rightarrow} F \rightarrow 0$ is exact if and only if $\varphi$ is surjective.

Let $E$ be a differential module with differential $d$, then by definition one has $\operatorname{Im}(d) \subset \operatorname{Ker}(d)$ so the non exactness of the sequence $E \stackrel{d}{\rightarrow} E \stackrel{d}{\rightarrow} E$ is measured by the module $H(E)=\operatorname{Ker}(d) / \operatorname{Im}(d)$ which is referred to as the homology of the differential module $E$. Let $\varphi: E \rightarrow F$ be a homomorphism of differential modules, then one has $\varphi(\operatorname{Im}(d)) \subset \operatorname{Im}(d)$ and $\varphi(\operatorname{Ker}(d)) \subset \operatorname{Ker}(d)$ (with an obvious abuse of notations) so $\varphi$ induces a homomorphism $\varphi_{*}: H(E) \rightarrow H(F)$ in homology. An important result for the computations of homology is given by the following proposition.

PROPOSITION 1. Let $0 \rightarrow E \stackrel{\varphi}{\rightarrow} F \stackrel{\psi}{\rightarrow} G \rightarrow 0$ be an exact sequence of differential modules; then there is a homomorphism $\partial: H(G) \rightarrow H(E)$ such that the triangle of homomorphisms

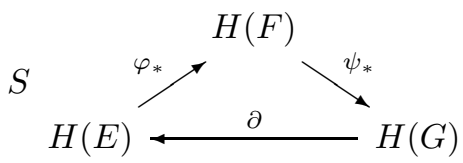

is exact.

The exactness at $H(F)$ is easy and we only sketch the construction of $\partial$. Let $z \in G$ be such that $d z=0$ and let us denote by $[z] \in H(G)$ the class of $z$. Since $\psi$ is surjective there is a $y \in F$ such that $\psi(y)=z$; one has $\psi(d y)=d \psi(y)=d z=0$ so $d y \in \operatorname{Ker}(\psi)$. By exactness at $F$, there is an $x \in E$ such that $\varphi(x)=d y$ and one has $\varphi(d x)=d \varphi(x)=d^{2} y=0$. Since $\varphi$ is injective it follows that $d x=0$ and we denote by $[x] \in H(E)$ the class of $x$. It turns out (and this is not difficult to verify) that $[x] \in H(E)$ does only depend on $[z] \in H(G)$ and that the mapping $[z] \mapsto \partial[z]=[x]$ is a homomorphism $\partial: H(G) \rightarrow H(E)$ which satisfies the statement of the proposition. 
Quite generally, a five terms exact sequence of the form

$$
0 \longrightarrow E \stackrel{\varphi}{\longrightarrow} F \stackrel{\psi}{\longrightarrow} G \longrightarrow 0
$$

is called a short exact sequence and given a short exact sequence of differential modules as in Proposition 1, the homomorphism $\partial: H(G) \rightarrow H(E)$ is called the connecting homomorphism of the short exact sequence of differential modules. The connecting homomorphism is natural (i.e. functorial) in the following sense: For any commutative diagram of differential modules

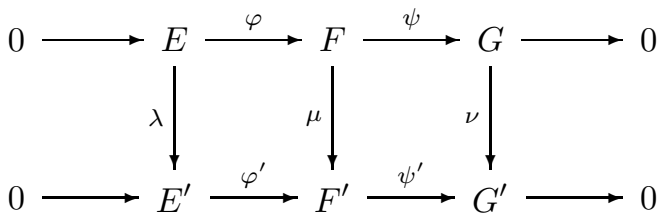

with exact rows, the diagram

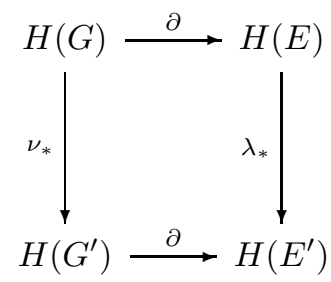

is commutative.

It is worth noticing here that although direct sums of differential modules are well defined, there is no natural tensor product of differential modules. A natural tensor product will be only obtained in the graded case, that is for complexes (see below).

In the case where $\mathbf{k}$ is a field, a differential module will be called a differential vector space or simply a differential space. In the examples connected with physics, $\mathbf{k}$ will always be either the field $\mathbb{R}$ or the field $\mathbb{C}$.

\section{Complexes}

By a complex, without other specification, we always mean a differential module $E$ which is $\mathbb{Z}$-graded, $E=\oplus E^{n}$, with a differential $d$ which is of degree 1 or -1 . When $d$ is of degree $-1, E$ is referred to as a chain complex and when $d$ is of degree $1, E$ is referred to as a cochain complex. One passes from the chain complexes to the cochain ones by changing the signs of the degrees $(n \mapsto-n)$. In the following we shall only consider the cochain case. The homology of a cochain complex $E$ is usually referred to as the cohomology of $E$. Since $d$ is homogeneous, the homology of a complex $E$ is $\mathbb{Z}$-graded : $H(E)=\bigoplus_{n \in \mathbb{Z}} H^{n}(E)$ with $H^{n}(E)=\operatorname{Ker}(d) \cap E^{n} / \operatorname{Im}(d) \cap E^{n}$. Many notions for complexes do only depend on the underlying $\mathbb{Z}_{2}$ graduation $\left(\mathbb{Z}_{2}=\mathbb{Z} / 2 \mathbb{Z}\right)$ so let us define a $\mathbb{Z}_{2}$-complex to be a differential module $E$ which is $\mathbb{Z}_{2}$-graded, $E=E^{0} \oplus E^{1}$, with a differential $d$ which is of degree $1(=-1)$. Again, the homology $H(E)$ of a $\mathbb{Z}_{2}$-complex is $\mathbb{Z}_{2}$-graded, that is $H(E)=H^{0}(E) \oplus H^{1}(E)$. A homomorphism of complexes or of $\mathbb{Z}_{2}$-complexes is 
a homomorphism of differential modules which is homogeneous of degree 0 .

Let $0 \longrightarrow E \stackrel{\varphi}{\longrightarrow} F \stackrel{\psi}{\longrightarrow} G \longrightarrow 0$ be a short exact sequence of cochain complexes; it follows from the definition of the connecting homomorphism $\partial$ that the exact triangle of Proposition 1 gives rise to the long exact sequence of homomorphisms

$$
\cdots \stackrel{\partial}{\longrightarrow} H^{n}(E) \stackrel{\varphi_{*}}{\longrightarrow} H^{n}(F) \stackrel{\psi_{*}}{\longrightarrow} H^{n}(G) \stackrel{\partial}{\longrightarrow} H^{n+1}(E) \stackrel{\varphi_{*}}{\longrightarrow} \cdots
$$

in cohomology. Similarily if $0 \longrightarrow E \stackrel{\varphi}{\longrightarrow} F \stackrel{\psi}{\longrightarrow} G \longrightarrow 0$ is a short exact sequence of $\mathbb{Z}_{2}$-complexes, the exact triangle of Proposition 1 gives rise to the exact hexagon of homomorphisms

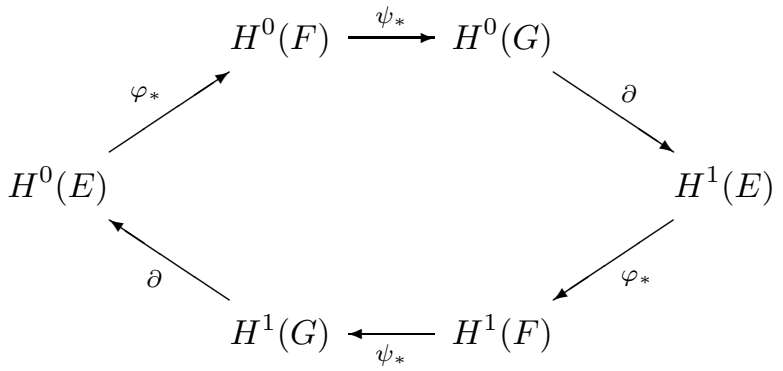

for the corresponding homologies.

Let $E$ and $F$ be two cochain complexes, (resp. $\mathbb{Z}_{2}$-complexes), their tensor product $E \otimes F$ is the graded module $E \otimes F=\oplus(E \otimes F)^{n}$ with $(E \otimes F)^{n}=$ $\underset{r+s=n}{\oplus} E^{r} \otimes F^{s}$ equipped with the differential $d$ defined by

$$
d(e \otimes f)=d e \otimes f+(-1)^{n} e \otimes d f,
$$

for any $e \in E^{n}$ and $f \in F$. One verifies that so defined on $E \otimes F, d$ is homogeneous of degree 1 and satisfies $d^{2}=0$ so that $E \otimes F$ is again a cochain complex, (resp. a $\mathbb{Z}_{2}$-complex). The virtue of this definition is the Künneth formula which we describe only for complexes of vector spaces in the following proposition, [36], [66].

PROPOSITION 2. Assume that the ring $\mathbf{k}$ is a field then one has $H(E \otimes F)=$ $H(E) \otimes H(F)$.

The above tensor product being the tensor product of graded vector spaces (over k) i.e. $H^{n}(E \otimes F)=\underset{r+s=n}{\oplus} H^{r}(E) \otimes H^{s}(F)$. This formula applies as well to the (co)chain complexes case and to the $\mathbb{Z}_{2}$-complexes case (whenever $\mathbf{k}$ is a field).

In the next section we shall describe a physical application of Proposition 2 combined with the remark that there is no such tensor product for differential spaces. We now achieve this section by the description of some classical constructions which will be used later.

Let $\mathfrak{g}$ be a Lie algebra, let $R$ be a representation space of $\mathfrak{g}$ and denote by $X \mapsto \pi(X) \in \operatorname{End}(R)$ the action of $\mathfrak{g}$ on $R$. An $R$-valued (Lie algebra) $n$-cochain of $\mathfrak{g}$ is a linear mapping $X_{1} \wedge \cdots \wedge X_{n} \mapsto \omega\left(X_{1}, \ldots, X_{n}\right)$ of $\wedge^{n} \mathfrak{g}$ into $R$. The vector space of these $n$-cochains will be denoted by $C_{\wedge}^{n}(\mathfrak{g}, R)$. One defines a homogeneous 
endomorphism $d$ of degree 1 of the $\mathbb{N}$-graded vector space $C_{\wedge}(\mathfrak{g}, R)=\oplus_{n} C_{\wedge}^{n}(\mathfrak{g}, R)$ of all $R$-valued cochains of $\mathfrak{g}$ by setting

$$
\begin{aligned}
& d(\omega)\left(X_{0}, \ldots, X_{n}\right)=\sum_{k=0}^{n}(-1)^{k} \pi\left(X_{k}\right) \omega\left(X_{0}, \stackrel{k}{.}, X_{n}\right) \\
& +\sum_{0 \leq r<s \leq n}(-1)^{r+s} \omega\left(\left[X_{r}, X_{s}\right], X_{0} \stackrel{\stackrel{r}{.} . \stackrel{s}{.} .}{ } . X_{n}\right)
\end{aligned}
$$

for $\omega \in C_{\wedge}^{n}(\mathfrak{g}, R)$ and $X_{i} \in \mathfrak{g}$. It follows from the Jacobi identity and from $\pi(X) \pi(Y)-\pi(Y) \pi(X)=\pi([X, Y])$ that $d^{2}=0$. Thus equipped with $d, C_{\wedge}(\mathfrak{g}, R)$ is a cochain complex and its cohomology, denoted by $H(\mathfrak{g}, R)$, is called the $R$-valued cohomology of $\mathfrak{g}$. The complexes $C_{\wedge}(\mathfrak{g}, R)$ are also called Chevalley-Eilenberg complexes and the differential $d$ is the Chevalley-Eilenberg differential.

There is a standard way to produce positive complexes (i.e. complexes $E=$ $\oplus E^{n}$ with $E^{n}=0$ for $n<0$ ) starting from (co)simplicial modules, (see e.g. 47], 66]). A pre-cosimplicial module (or semi-cosimplicial in the terminology of [66]) is a sequence of modules $\left(E^{n}\right)_{n \in \mathbb{N}}$ together with coface homomorphisms $\mathfrak{f}_{i}: E^{n} \rightarrow E^{n+1}, i \in\{0,1, \ldots, n+1\}$, satisfying

$$
\mathfrak{f}_{j} \mathfrak{f}_{i}=\mathfrak{f}_{i} \mathfrak{f}_{j-1} \quad \text { if } i<j \text {. }
$$

Given a pre-cosimplicial module $\left(E^{n}\right)$, one associates to it a positive complex $(E, d)$ by setting $E=\oplus_{n \in \mathbb{N}} E^{n}$ and $d=\sum_{i=0}^{n+1}(-1)^{i} \mathfrak{f}_{i}: E^{n} \rightarrow E^{n+1}$. One verifies that $d^{2}=0$ is implied by the coface relations $(\mathfrak{F})$. The differential $d$ will be referred to as the simplicial differential of $\left(E^{n}\right)$. The cohomology $H(E)=\oplus H^{n}(E)$ with $H^{n}(E)=\operatorname{Ker}\left(d: E^{n} \rightarrow E^{n+1}\right) / d E^{n-1}$ of $(E, d)$ will be referred to as the cohomology of the pre-cosimplicial module $\left(E^{n}\right)$. A cosimplicial module is a pre-cosimplicial module $\left(E^{n}\right)$ with coface homomorphisms $\mathfrak{f}_{i}$ as before together with codegeneracy homomorphisms $\mathfrak{s}_{i}: E^{n+1} \rightarrow E^{n}, i \in\{0, \ldots, n\}$, satisfying

and

$$
\mathfrak{s}_{j} \mathfrak{s}_{i}=\mathfrak{s}_{i} \mathfrak{s}_{j+1} \quad \text { if } i \leq j
$$

$$
\mathfrak{s}_{j} \mathfrak{f}_{i}= \begin{cases}\mathfrak{f}_{i} \mathfrak{s}_{j-1} & \text { if } i<j \\ I & \text { if } i=j \text { or } i=j+1 \\ \mathfrak{f}_{i-1} \mathfrak{s}_{j} & \text { if } i>j+1\end{cases}
$$

Given a cosimplicial module $\left(E^{n}\right)$ the elements $\omega$ of $E^{n}$ such that $s_{i}(\omega)=0$ for $i \in\{0, \cdots, n\}$ are called normalized cochains of degree $n$ and the graded module $N(E)=\underset{n}{\oplus} N^{n}(E)$ of all normalized cochains is a subcomplex of $E$ which has the same cohomology as the one of $E$, i.e. $H(E)$. The correspondence $\left(E^{n}\right) \mapsto N(E)$ defines an equivalence between the category of cosimplicial modules and the category of positive cochain complexes [66] which is referred to as the Dold-Kan correspondence (for the category of $\mathbf{k}$-modules).

Let $\mathcal{A}$ be an associative unital k-algebra and let $\mathcal{M}$ be an $(\mathcal{A}, \mathcal{A})$-bimodule. $\mathrm{A}$ $\mathcal{M}$-valued Hochschild cochain of degree $n$ or Hochschild $n$-cochain of $\mathcal{A}$ is a linear mapping $x_{1} \otimes \cdots \otimes x_{n} \mapsto \omega\left(x_{1}, \cdots, x_{n}\right)$ of $\otimes^{n} \mathcal{A}$ into $\mathcal{M}$. The $\mathbf{k}$-module of all $\mathcal{M}$ valued Hochschild $n$-cochains is denoted by $C^{n}(\mathcal{A}, \mathcal{M})$. The sequence $\left(C^{n}(\mathcal{A}, \mathcal{M})\right)$ is a cosimplicial module with cofaces $\mathfrak{f}_{i}$ and codegeneracies $\mathfrak{s}_{i}$ defined by [47, 66.

$\mathfrak{f}_{0}(\omega)\left(x_{0}, \ldots, x_{n}\right)=x_{0} \omega\left(x_{1}, \ldots, x_{n}\right)$

$\mathfrak{f}_{i}(\omega)\left(x_{0}, \ldots, x_{n}\right)=\omega\left(x_{0}, \ldots, x_{i-1} x_{i}, \ldots, x_{n}\right) \quad$ for $i \in\{1, \ldots, n\}$

$\mathfrak{f}_{n+1}(\omega)\left(x_{0}, \ldots, x_{n}\right)=\omega\left(x_{0}, \ldots, x_{n-1}\right) x_{n}$

and

$\mathfrak{s}_{i}(\omega)\left(x_{1}, \ldots, x_{n-1}\right)=\omega\left(x_{1}, \ldots, x_{i}, \mathbb{1}, x_{i+1}, \ldots, x_{n-1}\right) \quad$ for $i \in\{0, \ldots, n-1\}$ 
for $\omega \in C^{n}(\mathcal{A}, \mathcal{M})$ and $x_{i} \in \mathcal{A}$. The cohomology $H(\mathcal{A}, \mathcal{M})$ of this cosimplicial module is the $\mathcal{M}$-valued Hochschild cohomology of $\mathcal{A}$. In his case the simplicial differential is called the Hochschild differential.

There is a relation between the cohomology of a Lie algebra $\mathfrak{g}$ and the Hochschild cohomology of its universal enveloping algebra $U(\mathfrak{g})$ which we now describe again in the case where $\mathbf{k}$ is a field. Given a bimodule $\mathcal{M}$ over $U(\mathfrak{g})$ (that is a $(U(\mathfrak{g}), U(\mathfrak{g}))$-bimodule), let us define the representation $X \mapsto \operatorname{ad}(X)$ of $\mathfrak{g}$ in the vector space $\mathcal{M}$ by $\operatorname{ad}(X) m=X m-m X$ for $X \in \mathfrak{g}$ and $m \in \mathcal{M}$. Let $H\left(\mathfrak{g}, \mathcal{M}^{\text {ad }}\right)$ denote the Lie algebra cohomology of $\mathfrak{g}$ with values in $\mathcal{M}$ for the ad representation; its relation with the $\mathcal{M}$-valued Hochschild cohomology of $U(\mathfrak{g}), H(U(\mathfrak{g}), \mathcal{M})$ is given by the following theorem [7], [47].

THEOREM 1. Assume that $\mathbf{k}$ is a field, let $\mathfrak{g}$ be a Lie algebra over $\mathbf{k}$ and let $\mathcal{M}$ be a bimodule over $U(\mathfrak{g})$. Then there is a canonical isomorphism $H\left(\mathfrak{g}, \mathcal{M}^{\text {ad }}\right) \simeq$ $H(U(\mathfrak{g}), \mathcal{M})$.

If $R$ is a representation space of $\mathfrak{g}$ with action $X \mapsto \pi(X)$, then by the very definition of $U(\mathfrak{g}), \pi$ extends as a representation of $U(\mathfrak{g})$ so $R$ is canonically a left $U(\mathfrak{g})$-module. One converts $R$ into a $(U(\mathfrak{g}), U(\mathfrak{g}))$-bimodule $\mathcal{R}$ by acting on the right with the trivial action given by the counit of $U(\mathfrak{g})$ (recall that $U(\mathfrak{g})$ is a Hopf algebra); one then has $R=\mathcal{R}^{\text {ad }}$.

\section{A physical example: Naturality of ghosts}

The Wigner one-particle space for mass zero and spin one is the direct hilbertian integral $\int_{C_{+}} d \mu_{0}(p) \mathcal{H}(p)$ of 2-dimensional Hilbert spaces $\mathcal{H}(p)$ over the future light cone

$$
C_{+}=\left\{p \mid g^{\mu \nu} p_{\mu} p_{\nu}=p_{0}^{2}-\vec{p}^{2}=0, \quad p_{0}>0\right\}
$$

with respect to the invariant measure $d \mu_{0}(p)=\frac{1}{(2 \pi)^{3}} \frac{d^{3} \vec{p}}{2 p^{0}}$, where $\mathcal{H}(p)$ is the quotient of the subspace $\mathcal{Z}(p)=\left\{A_{\mu} \in \mathbb{C}^{4} \mid p^{\mu} A_{\mu}=0\right\}$ of $\mathcal{C}(p)=\mathbb{C}^{4}$ by the subspace $\mathcal{B}(p)=\left\{p_{\mu} \varphi \mid \varphi \in \mathbb{C}\right\}$ spanned by $p$, the scalar product of $\mathcal{H}(p)$ being induced by the indefinite scalar product of $\mathcal{C}(p)$ defined by $\left\langle A \mid A^{\prime}\right\rangle=-g^{\mu \nu} \bar{A}_{\mu} A_{\nu}^{\prime}$. The scalar product of $\mathcal{C}(p)$ is positive semi-definite on $\mathcal{Z}(p)$ and $\mathcal{B}(p)$ is its isotropic subspace whereas $\mathcal{Z}(p)$ is the orthogonal of $\mathcal{B}(p)$ in $\mathcal{C}(p)$. Notice that the indefinite metric space $\mathcal{C}(p)$ does not depend on $p$; we keep the reference to $p$ in order to remember that it carries a representation of the little group at $p$. The little group at $p$ here means the subgroup $\mathcal{L}_{p}$ of the Lorentz group which consists of the Lorentz tranformations $\Lambda$ preserving the (quadri) vector $p$, that is

$$
\mathcal{L}_{p}=\left\{\Lambda \in G L(4, \mathbb{R}) \mid \Lambda_{\lambda}^{\mu} \Lambda_{\rho}^{\nu} g^{\lambda \rho}=g^{\mu \nu} \text { and } \Lambda_{\nu}^{\mu} p^{\nu}=p^{\mu}\right\} .
$$

The occurrence of such a triplet $(\mathcal{C}(p), \mathcal{Z}(p), \mathcal{B}(p))$ where $\mathcal{C}(p)$ has an indefinite scalar product with $\mathcal{B}(p)$ isotropic having $\mathcal{Z}(p)$ as orthogonal, etc. is familiar in connection with indecomposable representations of groups (here the little group) [51], [1] and the indefinite metric is furthermore required to get a local covariant description of the electromagnetic gauge potential [61, [62], see also [46] in this context.

Let $Q(p)=Q$ be the linear endomorphism of $\mathcal{C}(p)$ defined by $Q(A)_{\mu}=p_{\mu} p^{\nu} A_{\nu}$. Then $Q$ is hermitian, i.e. $\left\langle A \mid Q A^{\prime}\right\rangle=\left\langle Q A \mid A^{\prime}\right\rangle$, and one has $Q^{2}=0$ in view of $p_{\mu} p^{\mu}=0$. Furthermore the image of $Q$ is $\mathcal{B}(p)$ and its kernel is $\mathcal{Z}(p)$. In other 
words $(\mathcal{C}(p), Q(p))$ is a differential space and $\mathcal{H}(p)$ is its homology, i.e. one has $\mathcal{H}(p)=\operatorname{Ker}(Q) / \operatorname{Im}(Q)$. Thus, apart from questions of domain and function spaces, everything is perfect at the "one-particle" level: Namely one has an indefinite metric space $\mathcal{C}$ which consists of functions $p \mapsto A_{\mu}(p) \in \mathcal{C}(p)$ on the light cone $C_{+}$and which is equipped with a differential $Q$ (i.e. an endomorphism satisfying $Q^{2}=0$ ) such that the physical one-particle space, (i.e. the Wigner space), is the homology $\operatorname{Ker}(Q) / \operatorname{Im}(Q)$ of $\mathcal{C}$.

As is well known, the role of $\mathcal{C}$ is to provide, via the Fock space constructions, an indefinite metric space on which the local covariant gauge potential (free) field operator acts; the corresponding space of physical states being of course the Fock space constructed over the one-particle Wigner space. However it turns out that the above one-particle (homological) picture does not generalize naively at the $n$ particle level for $n \geq 2$. To show what is involved here, let us analyze the situation at the two-particle level. In order to avoid complications connected with the problem of the choice of the function space and with the problem of symmetrization, let us work at fixed momenta $p_{1}$ and $p_{2}$ on the light cone $C_{+}$with $p_{1} \neq p_{2}$. The indefinite metric space is then the 16-dimensional space $\mathcal{C}\left(p_{1}\right) \otimes \mathcal{C}\left(p_{2}\right)$ whereas the space of physical states is the 4-dimensional Hilbert space $\mathcal{H}\left(p_{1}\right) \otimes \mathcal{H}\left(p_{2}\right)$. The point now is that there is no canonical way to construct $\mathcal{H}\left(p_{1}\right) \otimes \mathcal{H}\left(p_{2}\right)$ from $\mathcal{C}\left(p_{1}\right) \otimes \mathcal{C}\left(p_{2}\right)$. More precisely there is no canonical way to build a differential on $\mathcal{C}\left(p_{1}\right) \otimes \mathcal{C}\left(p_{2}\right)$ out of the differentials $Q\left(p_{1}\right)$ and $Q\left(p_{2}\right)$ of $\mathcal{C}\left(p_{1}\right)$ and $\mathcal{C}\left(p_{2}\right)$ in such a way that its homology is $\mathcal{H}\left(p_{1}\right) \otimes \mathcal{H}\left(p_{2}\right)$. In fact the most natural candidate would be $Q_{12}=Q\left(p_{1}\right) \otimes \operatorname{Id}_{2}+\operatorname{Id}_{1} \otimes Q\left(p_{2}\right)$ but this is not of square zero, only its third power vanishes, (for the "n-particle" case it would be the $(n+1)$-th power). Notice that with $Q_{12}$ satisfying $\left(Q_{12}\right)^{3}=0$ one can associate the generalized homologies (see below) $H_{(1)}\left(Q_{12}\right)=\operatorname{Ker}\left(Q_{12}\right) / \operatorname{Im}\left(\left(Q_{12}\right)^{2}\right)$ and $H_{(2)}\left(Q_{12}\right)=\operatorname{Ker}\left(\left(Q_{12}\right)^{2}\right) / \operatorname{Im}\left(Q_{12}\right)$ however it is easy to show that one canonically has $H_{(1)}\left(Q_{12}\right)=\mathcal{Z}\left(p_{1}\right) \otimes \mathcal{Z}\left(p_{2}\right)$ and that $H_{(2)}\left(Q_{12}\right)$ is isomorphic to $H_{(1)}\left(Q_{12}\right)$. Thus $H_{(1)}\left(Q_{12}\right)$ is a subspace of $\mathcal{C}\left(p_{1}\right) \otimes \mathcal{C}\left(p_{2}\right)$ on which the metric is positive semi-definite but it is still not the physical space $\mathcal{H}\left(p_{1}\right) \otimes \mathcal{H}\left(p_{2}\right)$.

Notice that we do not claim that there is no differential on $\mathcal{C}\left(p_{1}\right) \otimes \mathcal{C}\left(p_{2}\right)$ such that the corresponding homology is $\mathcal{H}\left(p_{1}\right) \otimes \mathcal{H}\left(p_{2}\right)$ but that we claim that there is no canonical one, that is no reasonable expression for such a differential in terms of the differentials $Q\left(p_{1}\right)$ and $Q\left(p_{2}\right)$. We refer to Appendix A for the precise statement.

As pointed out above, the origin of the difficulty is the non-existence of a good tensor product between differential spaces, i.e. between vector spaces equipped with endomorphisms of square zero. If instead of differential spaces one has complexes (of vector spaces), then the situation is much better; namely one has a canonical tensor product of complexes which is such that the homology of the tensor product is the tensor product of the homologies, (see last section). Furthermore one can show that the symmetrization-antisymmetrization involved in the Fock space construction does not spoil this picture.

Fortunately there is a canonical way (related to Theorem 4) to construct a complex $C(p)=C^{-1}(p) \oplus C^{0}(p) \oplus C^{1}(p)$ with a differential of degree 1 such that $C^{0}(p)=\mathcal{C}(p)$ and such that its $(c o)$ homology is again $\mathcal{H}(p)$. We now describe this 
construction. Let $\varepsilon^{\mu}$ be the (real) canonical base of $\mathcal{C}(p)=C^{0}(p)=\mathbb{C}^{4}$ and let $\omega^{(+)}$and $\omega^{(-)}$be the basis of the one dimensional spaces $C^{1}(p)$ and $C^{-1}(p)(\cong \mathbb{C})$. Define the homogeneous linear endomorphism $\delta(p)=\delta$ of degree 1 of $C(p)$ by $\delta \omega^{(+)}=0, \delta \varepsilon^{\mu}=\alpha p^{\mu} \omega^{(+)}$and $\delta \omega^{(-)}=p_{\mu} \varepsilon^{\mu},(\alpha$ being a non-vanishing constant $)$. It is clear that $\delta^{2}=0$ and it is straightforward to verify that the (co)homology $H(C(p))=\operatorname{Ker}(\delta) / \operatorname{Im}(\delta)$ of $C(p)$ is given by $H(C(p))=H^{0}(C(p))=\mathcal{H}(p)$. Notice that if $c \omega^{(-)}+A_{\mu} \varepsilon^{\mu}+\tilde{c} \omega^{(+)}$is an arbitrary element of $C(p), \delta$ reads in components $\delta A_{\mu}=p_{\mu} c, \delta c=0$ and $\delta \tilde{c}=\alpha p^{\lambda} A_{\lambda}$. One defines an indefinite hermitian scalar product on $C(p)$ extending the one of $C^{0}(p)=\mathcal{C}(p)$ for which $\delta$ is hermitian by set$\operatorname{ting}\left\langle\varepsilon^{\mu} \mid \varepsilon^{\nu}\right\rangle=-g^{\mu \nu},\left\langle\omega^{(+)} \mid \varepsilon^{\mu}\right\rangle=0,\left\langle\omega^{(+)} \mid \omega^{(+)}\right\rangle=0,\left\langle\omega^{(-)} \mid \varepsilon^{\mu}\right\rangle=0,\left\langle\omega^{(-)} \mid \omega^{(-)}\right\rangle=0$ and $\left\langle\omega^{(-)} \mid \omega^{(+)}\right\rangle=-\alpha^{-1}$. One can now construct the generalized Fock space $\mathfrak{F}(C)$ over the graded space $C$ of "functions" $p \mapsto\left(\tilde{c}(p), A_{\mu}(p), c(p)\right) \in C(p)$ on the future light cone. The space $\mathfrak{F}(C)$ is the graded-commutative algebra (freely) generated by the graded vector space $C$ and one extends $\delta$ as an antiderivation of $\mathfrak{F}(C)$, again denoted by $\delta$, which still satisfies $\delta^{2}=0$. The scalar product of $C$ extends canonically into an indefinite scalar product of $\mathfrak{F}(C)$ for which $\delta$ is hermitian and the cohomology $H^{0}(\delta)$ is (a dense subspace of) the physical space (i.e. the Fock space over the Wigner one-particle space). One then constructs as usual the local gauge potential field operator corresponding to the above one-particle $A_{\mu}$ as well as the fermionic ghost and antighost field operators corresponding to the above one-particle $c$ and $\tilde{c}$. In order that the ghost and the antighost fields be relatively local, it is necessary to take $\alpha$ purely imaginary, i.e. $\alpha=i \lambda$ with $\lambda \in \mathbb{R}_{*}$, otherwise one would obtain a factor $D^{(1)}$ in their anticommutators. With this choice $\left(\alpha=i \lambda, \lambda \in \mathbb{R}_{*}\right)$ the gauge potential, the ghost and the antighost field operators are local and relatively local, (see e.g. in [50]). Moreover these fields are hermitian by their very definition.

Let us say a few words on the case of spin two (and zero mass). In this case, the Wigner one-particle space is again the direct hilbertian integral $\int_{C_{+}} d \mu_{0}(p) \mathcal{H}(p)$ of two-dimensional Hilbert spaces $\mathcal{H}(p)$ over the future light cone with respect to $d \mu_{0}$ with $\mathcal{H}(p)=\mathcal{Z}(p) / \mathcal{B}(p)$ and $\mathcal{Z}(p) \subset \mathcal{C}(p)$ as above but now, $\mathcal{C}(p)$ is the 10-dimensional space of symmetric tensors $h_{\mu \nu}=h_{\nu \mu}$,

$$
\begin{aligned}
& \mathcal{Z}(p)=\left\{h_{\mu \nu} \in \mathcal{C}(p) \mid p^{\mu}\left(h_{\mu \nu}-\frac{1}{2} g_{\mu \nu} g^{\alpha \beta} h_{\alpha \beta}\right)=0\right\} \\
& \mathcal{B}(p)=\left\{p_{\mu} \varphi_{\nu}+p_{\nu} \varphi_{\mu} \mid \varphi_{\lambda} \in \mathbb{C}^{4}\right\}
\end{aligned}
$$

and the scalar product of $\mathcal{H}(p)$ is induced by the indefinite scalar product of $\mathcal{C}(p)$ defined by $\left\langle h \mid h^{\prime}\right\rangle=g^{\mu \nu} g^{\lambda \rho} \bar{h}_{\mu \lambda} h_{\nu \rho}^{\prime}-\frac{1}{2} g^{\alpha \beta} \bar{h}_{\alpha \beta} g^{\gamma \delta} h_{\gamma \delta}^{\prime}$. Again $\mathcal{B}(p)$ is a completely isotropic (4-dimensional) subspace of $\mathcal{C}(p)$ whereas the 6-dimensional space $\mathcal{Z}(p)$ is its orthogonal in $\mathcal{C}(p),\left(\mathcal{Z}(p)=\mathcal{B}(p)^{\perp}\right)$. It is worth noticing here that, apart from a multiplicative constant, the scalar product $\left\langle h \mid h^{\prime}\right\rangle$ is the unique non-trivial covariant scalar product on $\mathcal{C}(p)$ for which $\mathcal{B}(p)$ is isotropic; equivalently, the condition $p^{\mu}\left(h_{\mu \nu}-\frac{1}{2} g_{\mu \nu} g^{\alpha \beta} h_{\alpha \beta}\right)=0$ is the unique covariant linear (gauge) condition preserved by the translations of $\mathcal{B}(p)$. In view of the connection between the classical linearized gravity theory and the massless spin two particle, it is natural to interpret $h_{\mu \nu} \in \mathcal{C}(p)$ as the positive frequency part of the Fourier transform at $p$ of a perturbation $\underline{g}_{\mu \nu}(x)=g_{\mu \nu}+\varepsilon h_{\mu \nu}(x)$ of the Minkowskian metric $g_{\mu \nu}$. Translations by $\mathcal{B}(p)$ then read $h_{\mu \nu}(x) \mapsto h_{\mu \nu}(x)+\partial_{\mu} \varphi_{\nu}(x)+\partial_{\nu} \varphi_{\mu}(x)$ which corresponds 
to the first order in $\varepsilon$ (i.e. the linearization) of the action of infinitesimal diffeomorphisms (i.e. vector fields) whereas the condition to be in $\mathcal{Z}(p)$ translates into $\partial^{\mu}\left(h_{\mu \nu}(x)-\frac{1}{2} g_{\mu \nu} g^{\alpha \beta} h_{\alpha \beta}(x)\right)=0$ which is the first order in $\varepsilon$ of the de Donder harmonic coordinates condition $\frac{1}{\sqrt{|\underline{g}|}} \partial_{\mu}\left(\sqrt{|\underline{g}|} \underline{g}^{\mu \nu}\right)=\Delta_{\underline{g}}\left(x^{\nu}\right)=0$. It may well be that this observation (i.e. connection between Poincaré covariant Wigner analysis and de Donder harmonic coordinates condition) is a little more than a curiosity. In any case, we can now proceed as for the spin one case. One defines the graded vector space $C(p)=C^{-1}(p) \oplus C^{0}(p) \oplus C^{1}(p)$ by $C^{0}(p)=\mathcal{C}(p)$ and $C^{-1}(p) \simeq \mathbb{C}^{4} \simeq C^{1}(p)$ and we let $\omega^{(-) \mu}$ and $\omega^{(+) \mu}$ be the basis of $C^{-1}(p)$ and $C^{+1}(p)$ corresponding to the canonical base $\varepsilon^{\mu}$ of $\mathbb{C}^{4}$ and $\varepsilon^{\mu \nu}=\frac{1}{2}\left(\varepsilon^{\mu} \otimes \varepsilon^{\nu}+\varepsilon^{\nu} \otimes \varepsilon^{\mu}\right)$ be the corresponding basis of $C^{0}(p)=\mathcal{C}(p)$. One defines then a differential $\delta$ of degree 1 of $C(p)$ by setting $\delta \omega^{(+) \mu}=0, \delta \varepsilon^{\mu \nu}=\alpha\left(p^{\mu} \omega^{(+) \nu}+p^{\nu} \omega^{(+) \mu}\right)$ and $\delta \omega^{(-) \mu}=p_{\nu}\left(\varepsilon^{\mu \nu}-\frac{1}{2} g^{\mu \nu} g_{\alpha \beta} \varepsilon^{\alpha \beta}\right)$, $\alpha \in \mathbb{C}_{*}$. Again one verifies that the cohomology $H(C(p))=\operatorname{Ker}(\delta) / \operatorname{Im}(\delta)$ of $C(p)$ is given by $H(C(p))=H^{0}(C(p))=\mathcal{H}(p)$. If we let $c_{\rho} \omega^{(-) \rho}+h_{\mu \nu} \varepsilon^{\mu \nu}+\tilde{c}_{\lambda} \omega^{(+) \lambda}$ be an arbitrary element of $C(p), \delta$ reads in components $\delta h_{\mu \nu}=p_{\mu} c_{\nu}+p_{\nu} c_{\mu}, \delta c_{\mu}=0$ and $\delta \tilde{c}_{\mu}=\alpha p^{\nu}\left(h_{\mu \nu}-\frac{1}{2} g_{\mu \nu} g^{\alpha \beta} h_{\alpha \beta}\right)$. Finally, one defines an indefinite hermitian scalar product on $C(p)$ extending the one of $C^{0}(p)=\mathcal{C}(p)$ for which $\delta$ is hermitian by setting $\left\langle\varepsilon^{\lambda \rho} \mid \varepsilon^{\mu \nu}\right\rangle=\frac{1}{2}\left(g^{\lambda \mu} g^{\rho \nu}+g^{\lambda \nu} g^{\rho \mu}-g^{\lambda \rho} g^{\mu \nu}\right),\left\langle\omega^{(+) \lambda} \mid \varepsilon^{\mu \nu}\right\rangle=0,\left\langle\omega^{(+) \mu} \mid \omega^{(+) \nu}\right\rangle=0$, $\left\langle\omega^{(-) \lambda} \mid \varepsilon^{\mu \nu}\right\rangle=0,\left\langle\omega^{(-) \mu} \mid \omega^{(-) \nu}\right\rangle=0$ and $\left\langle\omega^{(-) \mu} \mid \omega^{(+) \nu}\right\rangle=\frac{1}{2 \alpha} g^{\mu \nu}$. Thus, apart from numbers of components, everything works as in the case of spin one, in particular one must again take $\alpha=i \lambda$ with $\lambda \in \mathbb{R}_{*}$ in order to have locality and relative locality between the hermitian free fields corresponding to $h_{\mu \nu}, c_{\lambda}$ and $\tilde{c}_{\rho}$.

The main message of this section is "the natural necessity" of ghosts (i.e. of graduations) in order to have a canonical local construction over the physical space and the fact that, in the previous examples (and others), there is a canonical way to introduce their counterpart at the one-particle level. This rewriting of the free field theory for zero mass and spin $\geq 1$ is certainly needed in order to start to introduce consistently interactions between abelian gauge fields. In particular this reformulation can be considered as the zero-step for the perturbative construction of quantum operatorial Yang-Mills theory.

\section{5. $N$-differential modules}

In the following, $N$ is a positive integer with $N \geq 2$. A module $E$ equipped with an endomorphism $d$ satisfying $d^{N}=0$ will be referred to as an $N$-differential module and the endomorphism $d$ as its $N$-differential. With this terminology, a 2-differential module is just a differential module.For each integer $m$ with $1 \leq m \leq N-1$, one defines the sub-modules $Z_{(m)}(E)$ and $B_{(m)}(E)$ by setting $Z_{(m)}(E)=\operatorname{Ker}\left(d^{m}\right)$ and $B_{(m)}(E)=\operatorname{Im}\left(d^{N-m}\right)$. It follows from the equation $d^{N}=0$ that $B_{(m)}(E)$ is a submodule of $Z_{(m)}(E)$ and the quotient modules $H_{(m)}(E)=Z_{(m)}(E) / B_{(m)}(E)$, $m \in\{1, \ldots, N-1\}$, will be referred to as the (generalized) homology of the $N$-differential module $E$.

Let $m$ be an integer with $1 \leq m \leq N-2$ and let $E$ be an $N$-differential module. One has the inclusions $Z_{(m)}(E) \subset Z_{(m+1)}(E)$ and $B_{(m)}(E) \subset B_{(m+1)}(E)$ which induces a homomorphism $[i]: H_{(m)}(E) \rightarrow H_{(m+1)}(E)$. One has also the inclusions $d Z_{(m+1)}(E) \subset Z_{(m)}(E)$ and $d B_{(m+1)}(E) \subset B_{(m)}(E)$ which induces a 
homomorphism $[d]: H_{(m+1)}(E) \rightarrow H_{(m)}(E)$. The following basic result show that the $H_{(m)}(E)$ are not independent [20], [14].

LEMMA 1. Let $\ell$ and $m$ be integers with $\ell \geq 1, m \geq 1$ and $\ell+m \leq N-1$. Then the following hexagon $\left(\mathcal{H}^{\ell, m}\right)$ of homomorphisms

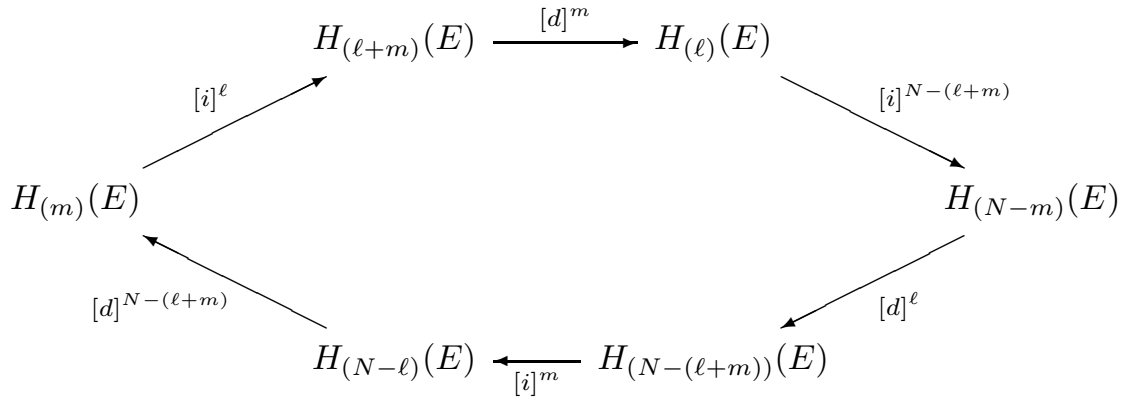

is exact.

One has obvious notions of homomorphism of $N$-differential modules, of $N$-differential submodule of an $N$-differential module, etc.. Let $\varphi: E \rightarrow E^{\prime}$ be a homomorphism of $N$-differential modules. Then one has $\varphi\left(Z_{(m)}(E)\right) \subset Z_{(m)}\left(E^{\prime}\right)$ and $\varphi\left(B_{(m)}(E)\right) \subset B_{(m)}\left(E^{\prime}\right)$ which implies that $\varphi$ induces a homomorphism $\varphi_{*}: H_{(m)}(E) \rightarrow H_{(m)}\left(E^{\prime}\right), \forall m \in\{1, \ldots, N-1\}$. Moreover $\varphi_{*}$ satisfies $\varphi_{*} \circ[i]=$ $[i] \circ \varphi_{*}$ and $\varphi_{*} \circ[d]=[d] \circ \varphi_{*}$. Proposition 1 has the following generalization for $N$-differential modules.

PROPOSITION 3. Let $0 \rightarrow E \stackrel{\varphi}{\rightarrow} F \stackrel{\psi}{\rightarrow} G \rightarrow 0$ be a short exact sequence of $N$-differential modules. Then there are homomorphisms $\partial: H_{(m)}(G) \rightarrow H_{(N-m)}(E)$ for $m \in\{1, \ldots, N-1\}$ such that the following hexagons $\left(\mathcal{H}_{n}\right)$ of homomorphisms

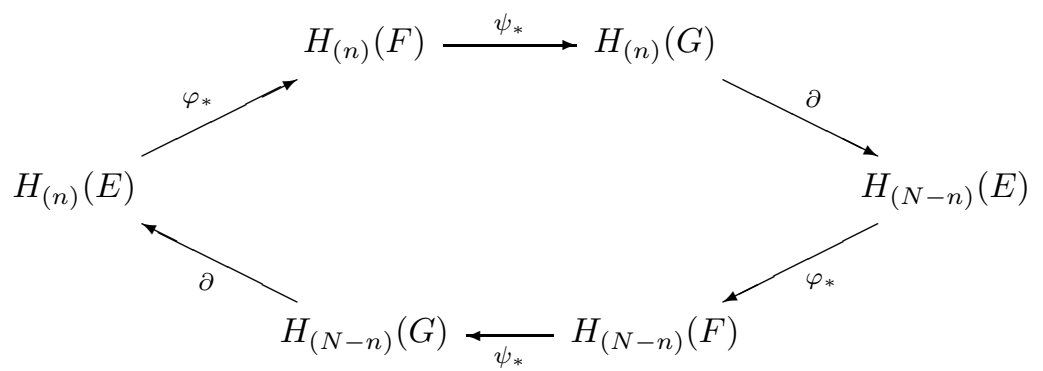

are exact, for $n \in\{1, \ldots, N-1\}$.

For a proof, we refer to [43], [14], 15]. In fact, there is a way to interpret $\left(\mathcal{H}_{n}\right)$ as the exact hexagon corresponding to a short exact sequence of $\mathbb{Z}_{2}$-complexes $0 \rightarrow C_{(n)}(E) \rightarrow C_{(n)}(F) \rightarrow C_{(n)}(G) \rightarrow 0$ associated with the $N$-complexes, [15].

Let us now give some criteria ensuring the vanishing of the $H_{(n)}(E)$. The first criterion is extracted from 40 .

LEMMA 2. Let $E$ be an $N$-differential module such that $H_{(k)}(E)=0$ for some integer $k$ with $1 \leq k \leq N-1$. Then one has $H_{(n)}(E)=0$ for any integer $n$ with $1 \leq n \leq N-1$. 
A short proof of this lemma using Lemma 1 is given in 14. The next criterion which is in 40 is connected with an appropriate generalization of homotopy, see in 43 and in 14. It is given by the following lemma the proof of which is easy.

LEMMA 3. Let $E$ be an $N$-differential module such that there are endomorphisms of modules $h_{k}: E \rightarrow E$ for $k=0,1, \ldots, N-1$ satisfying $\sum_{k=0}^{N-1} d^{N-1-k} h_{k} d^{k}=$ $I d_{E}$; then one has $H_{(n)}(E)=0$ for each integer $n$ with $1 \leq n \leq N-1$.

In order to formulate the last criterion, we recall the definition of $q$-numbers. With $q \in \mathbf{k}$, one associates a mapping []$_{q}: \mathbb{N} \rightarrow \mathbf{k}, n \mapsto[n]_{q}$, which is defined by setting $[0]_{q}=0$ and $[n]_{q}=1+\cdots+q^{n-1}=\sum_{k=0}^{n-1} q^{k}$ for $n \geq 1,\left(q^{0}=1\right)$. For $n \in \mathbb{N}$ with $n \geq 1$, one defines the $q$-factorial $[n]_{q} ! \in \mathbf{k}$ by $[n]_{q} \ldots 1=\prod_{k=1}^{n}[k]_{q}$. For integers $n$ and $m$ with $n \geq 1$ and $0 \leq m \leq n$, one defines inductively the $q$-binomial coefficients $\left[\begin{array}{c}n \\ m\end{array}\right]_{q} \in \mathbf{k}$ by setting $\left[\begin{array}{l}n \\ 0\end{array}\right]_{q}=\left[\begin{array}{l}n \\ n\end{array}\right]_{q}=1$ and $\left[\begin{array}{c}n \\ m\end{array}\right]_{q}+q^{m+1}\left[\begin{array}{c}n \\ m+1\end{array}\right]_{q}=\left[\begin{array}{c}n+1 \\ m+1\end{array}\right]_{q}$ for $0 \leq m \leq n-1$. As in 43 let us introduce the following assumptions $\left(A_{0}\right)$ and $\left(A_{1}\right)$ on the ring $\mathbf{k}$ and the element $q$ of $\mathbf{k}$ :

$\left(A_{0}\right) \quad[N]_{q}=0$

$\left(A_{1}\right) \quad[N]_{q}=0$ and $[n]_{q}$ is invertible for $1 \leq n \leq N-1,(n \in \mathbb{N})$.

Notice that $[N]_{q}=0$ implies that $q^{N}=1$ and therefore that $q$ is invertible. Furthermore if $q$ is invertible one has $[n]_{q^{-1}}=q^{-n+1}[n]_{q}, \forall n \in \mathbb{N}$. Therefore Assumption $\left(A_{0}\right)$, (resp. $\left.\left(A_{1}\right)\right)$, for $\mathbf{k}$ and $q \in \mathbf{k}$ is equivalent to Assumption $\left(A_{0}\right),\left(\operatorname{resp} .\left(A_{1}\right)\right)$, for $\mathbf{k}$ and $q^{-1} \in \mathbf{k}$. Let us give two typical examples:

1. $\mathbf{k}=\mathbb{C}, q \in \mathbb{C}$. Then Assumption $\left(A_{0}\right)$ means that $q$ is an $N$-th root of unity distinct of 1 and Assumption $\left(A_{1}\right)$ means that $q$ is a primitive $N$-th root of unity.

2. $\mathbf{k}=\mathbb{Z}_{N}=\mathbb{Z} / N \mathbb{Z}$, then $1 \in \mathbf{k}$ satisfies Assumption $\left(A_{0}\right)$ and Assumption $\left(A_{1}\right)$ is satisfied if and only if $N$ is a prime number.

A useful result is that if $\mathbf{k}$ and $q \in \mathbf{k}$ satisfy Assumption $\left(A_{1}\right)$ then one has $\left[\begin{array}{l}N \\ m\end{array}\right]_{q}=0$ for $m \in\{1, \ldots, N-1\}$; notice that Assumption $\left(A_{0}\right)$ is not sufficient in order to have this result.

We are now ready to state the last criterion [13].

LEMMA 4. Suppose that $\mathbf{k}$ and $q \in \mathbf{k}$ satisfy $\left(A_{1}\right)$ and let $E$ be an $N$ differential module. Assume that there is a module-endomorphism $h$ of $E$ such that $h d-q d h=I d_{E}$. Then one has $H_{(n)}(E)=0$ for each integer $n$ with $1 \leq n \leq N-1$.

In order to proof this lemma, one shows that in the unital k-algebra generated by $h$ and $d$ with the relation $h d-q d h=\mathbb{1}$ one has $\sum_{k=0}^{N-1} d^{N-1-k} h^{N-1} d^{k}=[N-1]_{q} ! \mathbb{1}$, which implies the result in view of Lemma 3 since $[N-1]_{q}$ ! is invertible in $\mathbf{k}$ (see in 43 and in 14 ). 
It is obvious that Lemma 4 above is closely related to the theory of $q$-oscillators, (e.g. $d$ corresponds to the creation operator whereas $h$ corresponds to the annihilation operator), and this is the essence of the proof of [14. As well known in physics, there is another natural way to produce creation operators with vanishing $N$-th powers which consists in considering parafermions of order $N-1$; this has the generalization we now describe.

As already pointed out (in Section 2 and Section 4) there is no natural tensor product between differential modules. The same is true for $N$-differential modules with $N$ fixed. However, if $\left(E^{\prime}, d^{\prime}\right)$ is an $N^{\prime}$-differential module and if $\left(E^{\prime \prime}, d^{\prime \prime}\right)$ is an $N^{\prime \prime}$-differential module $\left(N^{\prime}, N^{\prime \prime} \geq 2\right)$ then one defines an $\left(N^{\prime}+N^{\prime \prime}-1\right)$-differential $d$ on $E^{\prime} \otimes E^{\prime \prime}$ by setting

$$
d=d^{\prime} \otimes I^{\prime \prime}+I^{\prime} \otimes d^{\prime \prime}
$$

where $I^{\prime}$ (resp. $I^{\prime \prime}$ ) denotes the identity mapping $I d_{E^{\prime}}$ (resp. $I d_{E^{\prime \prime}}$ ) of $E^{\prime}$ (resp. of $\left.E^{\prime \prime}\right)$. Therefore, a natural construction of an $N$-differential module consists in starting with $(N-1)$ ordinary differential modules $\left(E_{i}, d_{i}\right)$ and equipping their tensor product $E=E_{1} \otimes \cdots \otimes E_{N-1}$ with the $N$-differential

$$
d=d_{1} \otimes I_{2} \otimes \cdots \otimes I_{N-1}+\cdots+I_{1} \otimes \cdots \otimes I_{N-2} \otimes d_{N-1} .
$$

If all the $\left(E_{i}, d_{i}\right)$ are identical, with $d_{i}$ being a fermionic creation operator, the above formula is the Green ansatz [34] for the parafermionic creation operator of order $N-1$.

In the case where $\mathbf{k}$ is a field, an $N$-differential module will be referred to as an $N$-differential vector space. Assume that $E$ is a finite-dimensional $N$-differential vector space. Then one has $E \simeq \operatorname{Ker}\left(d^{n}\right) \oplus \operatorname{Im}\left(d^{n}\right)=Z_{(n)}(E) \oplus B_{(N-n)}(E)$ and $E \simeq \operatorname{Ker}\left(d^{N-n}\right) \oplus \operatorname{Im}\left(d^{N-n}\right)=Z_{(N-n)}(E) \oplus B_{(n)}(E)$ which together with $Z_{(n)}(E) \simeq B_{(n)}(E) \oplus H_{(n)}(E)$ and $Z_{(N-n)}(E) \simeq B_{(N-n)}(E) \oplus H_{(N-n)}(E)$ implies (since $\operatorname{dim}(E)<\infty$ ) that $H_{(n)}(E)$ and $H_{(N-n)}(E)$ are isomorphic. In the case where $E$ is a finite-dimensional $N$-differential vector space over $\mathbf{k}=\mathbb{R}$ or $\mathbb{C}$, one can show (see e.g. in [35) by decomposing $E$ into indecomposable factor for the action of the $N$-differential $d$ that one has an isomorphism $E \simeq \oplus_{n=1}^{N} \mathbf{k}^{n} \otimes \mathbf{k}^{m_{n}}$, $d \simeq \oplus_{n=2}^{N} D_{n} \otimes I d_{\mathbf{k}^{m_{n}}}$ with

$$
D_{n}=\left(\begin{array}{ccccccc}
0 & 1 & 0 & . & . & . & 0 \\
\cdot & . & . & . & & & \cdot \\
\cdot & & \cdot & \cdot & . & & \cdot \\
\cdot & & & \cdot & \cdot & \cdot & . \\
\cdot & & & & \cdot & \cdot & 0 \\
. & & & & & \cdot & 1 \\
0 & . & . & . & . & . & 0
\end{array}\right) \in M_{n}(\mathbf{k})
$$

where the multiplicities $m_{n}, n \in\{1, \ldots, N\}$, are invariants of $(E, d)$ with $\sum_{n=1}^{N} n m_{n}=\operatorname{dim}(E)$. Notice that one has $m_{N} \geq 1$ whenever $d^{N-1} \neq 0$. Notice also that the above decomposition of $d$ is its Jordan normal-form. In terms of the multiplicities, one can easily compute the dimensions of the vector spaces $H_{(k)}(E)$. The result is given by the following proposition. 
PROPOSITION 4. Let $E$ be a finite dimensional $N$-differential vector space over $\mathbb{R}$ or $\mathbb{C}$ with multiplicities $m_{n}, n \in\{1,2, \ldots, N\}$, then one has for each integer $k$ with $1 \leq k \leq N / 2$

$$
\operatorname{dim} H_{(k)}(E)=\operatorname{dim} H_{(N-k)}(E)=\sum_{j=1}^{k} \sum_{i=j}^{N-j} m_{i}
$$

Although easy, that kind of results is useful for applications (see below).

\section{N-complexes}

An $N$-complex of modules 40 or simply an $N$-complex is an $N$-differential module $E$ which is $\mathbb{Z}$-graded, i.e. $E=\oplus_{n \in \mathbb{Z}} E^{n}$, with a homogeneous $N$-differential $d$ of degree 1 or -1 . When $d$ is of degree 1 then $E$ is referred to as a cochain $N$-complex and when $d$ is of degree -1 then $E$ is referred to as a chain $N$-complex. Here we adopt the cochain language and therefore in the following an $N$-complex, without other specification, always means a cochain $N$-complex of modules. If $E$ is an $N$-complex then the $H_{(m)}(E)$ are $\mathbb{Z}$-graded modules; $H_{(m)}(E)=\oplus_{n \in \mathbb{Z}} H_{(m)}^{n}(E)$ with $H_{(m)}^{n}(E)=\operatorname{Ker}\left(d^{m}: E^{n} \rightarrow E^{n+m}\right) / d^{N-m}\left(E^{n+m-N}\right)$. In this case the hexagon $\left(\mathcal{H}^{\ell, m}\right)$ of Lemma 1 splits into long exact sequences $\left(\mathcal{S}_{p}^{\ell, m}\right), p \in \mathbb{Z}$

$$
\begin{aligned}
& \cdots \rightarrow H_{(m)}^{N r+p}(E) \stackrel{[i]^{\ell}}{\longrightarrow} H_{(\ell+m)}^{N r+p}(E) \stackrel{[d]^{m}}{\longrightarrow} H_{(\ell)}^{N r+p+m}(E) \\
&\left(\mathcal{S}_{p}^{\ell, m}\right) \stackrel{[i]^{N-(\ell+m)}}{\longrightarrow} H_{(N-m)}^{N r+p+m}(E) \stackrel{[d]^{\ell}}{\longrightarrow} H_{(N-(\ell+m))}^{N r+p+\ell+m}(E) \\
& \stackrel{[i]^{m}}{\longrightarrow} H_{(N-\ell)}^{N r+p+\ell+m}(E) \stackrel{[d]^{N-(\ell+m)}}{\longrightarrow} H_{(m)}^{N(r+1)+p}(E) \stackrel{[i]^{\ell}}{\longrightarrow} \ldots
\end{aligned}
$$

One has $\left(\mathcal{S}_{p}^{\ell, m}\right)=\left(\mathcal{S}_{p+N}^{\ell, m}\right)$

Let $E$ and $E^{\prime}$ be $N$-complexes, a homomorphism of $N$-complexes of $E$ into $E^{\prime}$ is a homomorphism of $N$-differential modules $\varphi: E \rightarrow E^{\prime}$ which is homogeneous of degree 0, (i.e. $\left.\varphi\left(E^{n}\right) \subset E^{\prime n}\right)$. Such a homomorphism of $N$-complexes induces module-homomorphisms $\varphi_{*}: H_{(m)}^{n}(E) \rightarrow H_{(m)}^{n}\left(E^{\prime}\right)$ for $n \in \mathbb{Z}$ and $1 \leq m \leq N-1$.

Let $0 \rightarrow E \stackrel{\varphi}{\rightarrow} F \stackrel{\psi}{\rightarrow} G \rightarrow 0$ be a short exact sequence of $N$-complexes, then the hexagon $\left(\mathcal{H}_{n}\right)$ of Lemma 2 splits into long exact sequences $\left(\mathcal{S}_{n, p}\right), p \in \mathbb{Z}$

$$
\begin{aligned}
& \cdots \rightarrow H_{(n)}^{N r+p}(E) \stackrel{\varphi_{*}}{\longrightarrow} H_{(n)}^{N r+p}(F) \stackrel{\psi_{*}}{\longrightarrow} H_{(n)}^{N r+p}(G) \\
&\left(\mathcal{S}_{n, p}\right) \stackrel{\partial}{\rightarrow} H_{(N-n)}^{N r+p+n}(E) \stackrel{\varphi_{*}}{\longrightarrow} H_{(N-n)}^{N r+p+n}(F) \stackrel{\psi_{*}}{\longrightarrow} H_{(N-n)}^{N r+p+n}(G) \\
& \stackrel{\partial}{\rightarrow} H_{(n)}^{N(r+1)+p}(E) \stackrel{\varphi_{*}}{\longrightarrow} \ldots
\end{aligned}
$$

One has again $\left(\mathcal{S}_{n, p}\right)=\left(\mathcal{S}_{n, p+N}\right)$. 
In the following of this section, $\left(E^{n}\right)_{n \in \mathbb{N}}$ is a pre-cosimplicial module (see in Section 3), $E$ denotes the (positively) graded module $\oplus_{n} E^{n}$ and $q \in \mathbf{k}$ is such that $[N]_{q}=0$, i.e. such that $\mathbf{k}$ and $q \in \mathbf{k}$ satisfy the assumption $\left(A_{0}\right)$ of Section 5 . One can construct a sequence $\left(d_{n}\right)_{n \in \mathbb{N}}$ of $N$-differentials of degree 1 on $E$ by using $q \in \mathbf{k}$ as above [14. Here we shall only consider the first two $d_{0}$ and $d_{1}$ which are the most natural ones. They are defined by setting for $n \in \mathbb{N}$

$$
d_{0}=\sum_{i=0}^{n+1} q^{i} \mathfrak{f}_{i}: E^{n} \rightarrow E^{n+1}
$$

and

$$
d_{1}=\sum_{i=0}^{n} q^{i} \mathfrak{f}_{i}-q^{n} \mathfrak{f}_{n+1}: E^{n} \rightarrow E^{n+1}
$$

LEMMA 5. One has $d_{0}^{N}=0$ and $d_{1}^{N}=0$.

This is a consequence of $[N]_{q}=0$ and of the relations $(\mathfrak{F})$; for a proof we refer to 114 .

Thus $\left(E, d_{0}\right)$ and $\left(E, d_{1}\right)$ are $N$-complexes and, as shown in [14], there are natural homomorphisms of the cohomology $H(E)$ of the pre-cosimplicial module $\left(E^{n}\right)$ into the generalized cohomologies of these $\mathrm{N}$-complexes. In order to compute completely these generalized cohomologies we shall need some more assumptions. We shall need Assumption $\left(A_{1}\right)$ for $\mathbf{k}$ and $q \in \mathbf{k}$ and we shall restrict attention to cosimplicial modules. The generalized cohomologies of $\left(E, d_{0}\right)$ and $\left(E, d_{1}\right)$ are then given by the following theorem [14.

THEOREM 2. Let $\mathbf{k}$ and $q \in \mathbf{k}$ satisfy Assumption $\left(A_{1}\right)$ and let $\left(E^{n}\right)$ be a cosimplicial module. Then one has:

(0) $\quad H_{(m)}^{N r-1}\left(E, d_{0}\right)=H^{2 r-1}(E), H_{(m)}^{N(r+1)-m-1}\left(E, d_{0}\right)=H^{2 r}(E)$ and $H_{(m)}^{n}\left(E, d_{0}\right)=0$ otherwise,

(1) $\quad H_{(m)}^{N r}\left(E, d_{1}\right)=H^{2 r}(E), H_{(m)}^{N(r+1)-m}\left(E, d_{1}\right)=H^{2 r+1}(E)$ and $H_{(m)}^{n}\left(E, d_{1}\right)=0$ otherwise,

for $r \in \mathbb{N}$ and $m \in\{1, \ldots, N-1\}$.

There is of course a dual statement for simplicial modules and the analogs of $d_{0}$ and $d_{1}$ which are then of degree -1 , see in [14]. The above theorem and its dual version cover all the (co)simplicial cases investigated so far that I know (49, [20, [13], 143] and 14). In 14 the generalized cohomology of $E$ for every $d_{n}$ $(n \in \mathbb{N})$ was also computed in the case of a cosimplicial module $\left(E^{n}\right)$ as well as the generalized homologies of their chain analogs in the case of a simplicial module $\left(E_{n}\right)$ under assumption $\left(A_{1}\right)$ for $\mathbf{k}$ and $q \in \mathbf{k}$. As a rule, we found there that (in the (co)simplicial case) these generalized (co)homologies do only depend on the ordinary (co)homology of the (co)simplicial module. In fact one of the ingredients in the proof of the above theorem is to use the whole sequence of $N$-differentials $\left(d_{n}\right)_{n \in \mathbb{N}}$ because, for any $p \in \mathbb{N}$ there is a $n_{p} \in \mathbb{N}$ such that $d_{n}$ coincides with the simplicial differential in degree $r$ (i.e. on $E^{r}$ ) whenever $n \geq n_{p}$ and $r \leq p$; the proof 
is nevertheless highly non trivial, (see in [14]).

Many notions for $N$-complexes do only depend on the underlying $\mathbb{Z}_{N}$-graduation $\left(\mathbb{Z}_{N}=\mathbb{Z} / N \mathbb{Z}\right)$ so let us define a $\mathbb{Z}_{N}$-complex to be an $N$-differential module which

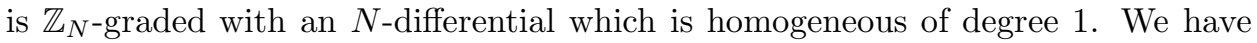
avoided the terminology $\mathbb{Z}_{N}-N$-complex since we shall not consider differential modules equipped with $\mathbb{Z}_{N}$ graduations with $N \geq 3$ and since a $\mathbb{Z}_{2}$-complex with the above definition is a $\mathbb{Z}_{2}$-complex according to the definition of Section 3 . We now give an example of $\mathbb{Z}_{N}$-complex.

Let $\mathbf{k}$ and $q \in \mathbf{k}$ satisfy Assumption $\left(A_{1}\right)$ and let us introduce the standard basis $E_{\ell}^{k},(k, \ell \in\{1, \ldots, N\})$, of the algebra $M_{N}(\mathbf{k})$ of $N \times N$ matrices defined by $\left(E_{\ell}^{k}\right)_{j}^{i}=\delta_{j}^{k} \delta_{\ell}^{i}$. One has $E_{\ell}^{k} E_{s}^{r}=\delta_{s}^{k} E_{\ell}^{r}$ and $\sum_{n=1}^{N} E_{n}^{n}=\mathbb{1}$. It follows that one can equip $M_{N}(\mathbf{k})$ with a structure of $\mathbb{Z}_{N}$-graded algebra, $M_{N}(\mathbf{k})=\oplus_{a \in \mathbb{Z}_{N}} M_{N}(\mathbf{k})^{a}$, by giving to $E_{\ell}^{k}$ the degree $k-\ell \bmod (N)$. Let $e=\lambda_{1} E_{1}^{2}+\cdots+\lambda_{N-1} E_{N-1}^{N}+\lambda_{N} E_{N}^{1}$ be an element of degree 1 of $M_{N}(\mathbf{k})$ and define the endomorphism $d$ by $d(A)=e A-q^{a} A e$ for $A \in M_{N}(\mathbf{k})^{a}$. One has $d^{N}=0$ so $\left(M_{N}(\mathbf{k}), d\right)$ is a $\mathbb{Z}_{N}$-complex. One verifies that $e^{N}=\lambda_{1} \ldots \lambda_{N} \mathbb{1}$ and that $e^{N-1} d(A)-q d\left(e^{N-1} A\right)=(1-q) \lambda_{1} \ldots \lambda_{N} A$. Therefore if $1-q$ and the $\lambda_{i}$ are invertible in $\mathbf{k}$, Lemma 4 implies that $H_{(n)}\left(M_{N}(\mathbf{k}), d\right)=0$ for $n \in\{1, \ldots, N-1\}$. It is worth noticing that the above $N$-differential satisfies the graded $q$-Leibniz rule $d(A B)=d(A) B+q^{a} A d(B), \forall A \in M_{N}(\mathbf{k})^{a}, \forall B \in M_{N}(\mathbf{k})$.

It is clear that for any $N$-complex one has an underlying $\mathbb{Z}_{N}$-complex which is obtained by retaining only the degree modulo $N$. On the other hand starting from a $\mathbb{Z}_{N}$-complex $E=\oplus_{n \in \mathbb{Z}_{N}} E^{n}$ like $\left(M_{N}(\mathbf{k}), d\right)$ above, one can construct an $N$-complex $\tilde{E}=\oplus_{n \in \mathbb{Z}} \tilde{E}^{n}$ by setting $\tilde{E}^{n}=E^{\pi(n)}$ where $\pi$ is the canonical projection of $\mathbb{Z}$ onto $\mathbb{Z}_{N}$, the definition of the $N$-differential on $\tilde{E}$ being obvious in terms of the one of $E$.

The content of Section 5 and Section 6 is based on [14 (see also in [20] and in 13]). Particular $N$-complexes were introduced and analysed in $\left[49\right.$ for $\mathbf{k}=\mathbb{Z}_{N}$, $(N$ prime). Several mathematicians wrote on $N$-complexes at the end of the 40 's, beginning of the 50's. The subject was reconsidered in 40 and developed more recently in [20], [13], 43] and 14]. In [43 an approach in the line of modern homological algebra [7] was developed with the introduction of generalizations of the functors Ext and Tor.

\section{N-complexes of tensor fields}

In this section we shall describe $N$-complexes of tensor fields on $\mathbb{R}^{D}$ which generalize the complex $\Omega\left(\mathbb{R}^{D}\right)$ of differential forms [17], [18]. Therefore here the ring $\mathbf{k}$ is the field $\mathbb{R}$ (or eventually $\mathbb{C}$ if one considers complex tensors). Furthermore, in such an $N$-complex, for each degree the tensor fields will be smooth mapping $x \mapsto T(x)$ of $\mathbb{R}^{D}$ into the vector space of covariant tensors of a given Young symmetry. Let us recall that this implies that the representation of $G L_{D}$ in the corresponding space of tensors is irreducible. For Young diagrams, etc. we refer to [30] and for more details and developments we refer to [17, 18. 
Throughout the following $\left(x^{\mu}\right)=\left(x^{1}, \ldots, x^{D}\right)$ denotes the canonical coordinates of $\mathbb{R}^{D}$ and $\partial_{\mu}$ are the corresponding partial derivatives which we identify with the corresponding covariant derivatives associated to the canonical flat linear connection of $\mathbb{R}^{D}$. Thus, for instance, if $T$ is a covariant tensor field of degree $p$ on $\mathbb{R}^{D}$ with components $T_{\mu_{1} \ldots \mu_{p}}(x)$, then $\partial T$ denotes the covariant tensor field of degree $p+1$ with components $\partial_{\mu_{1}} T_{\mu_{2} \ldots \mu_{p+1}}(x)$. The operator $\partial$ is a first-order differential operator which increases by one the tensorial degree.

In this context, the space $\Omega\left(\mathbb{R}^{D}\right)$ of differential forms on $\mathbb{R}^{D}$ is the graded vector space of (covariant) antisymmetric tensor fields on $\mathbb{R}^{D}$ with graduation induced by the tensorial degree whereas the exterior differential $d$ is the composition of the above $\partial$ with antisymmetrisation, i.e.

$$
d=\mathbf{A}_{p+1} \circ \partial: \Omega^{p}\left(\mathbb{R}^{D}\right) \rightarrow \Omega^{p+1}\left(\mathbb{R}^{D}\right)
$$

where $\mathbf{A}_{p}$ denotes the antisymmetrizer on tensors of degree $p$. One has $d^{2}=0$ and the Poincare lemma asserts that the cohomology of the complex $\left(\Omega\left(\mathbb{R}^{D}\right), d\right)$ is trivial, i.e. that one has $H^{p}\left(\Omega\left(\mathbb{R}^{D}\right)\right)=0, \forall p \geq 1$ and $H^{0}\left(\Omega\left(\mathbb{R}^{D}\right)\right)=\mathbb{R}$.

From the point of view of Young symmetry, antisymmetric tensors correspond to Young diagrams (partitions) described by one column of cells, i.e. the space of values of $p$-forms corresponds to one column of $p$ cells, $\left(1^{p}\right)$, whereas $\mathbf{A}_{p}$ is the associated Young symmetrizer, (see e.g. in [30]).

There is a relatively easy way to generalize the pair $\left(\Omega\left(\mathbb{R}^{D}\right), d\right)$ which we now describe. Let $Y=\left(Y_{p}\right)_{p \in \mathbb{N}}$ be a sequence of Young diagrams such that the number of cells of $Y_{p}$ is $p, \forall p \in \mathbb{N}$ (i.e. such that $Y_{p}$ is a partition of the integer $p$ for any $p)$. We define $\Omega_{Y}^{p}\left(\mathbb{R}^{D}\right)$ to be the vector space of smooth covariant tensor fields of degree $p$ on $\mathbb{R}^{D}$ which have the Young symmetry type $Y_{p}$ and we let $\Omega_{Y}\left(\mathbb{R}^{D}\right)$ be the graded vector space $\underset{p}{\oplus} \Omega_{Y}^{p}\left(\mathbb{R}^{D}\right)$. We then generalize the exterior differential by setting $d=\mathbf{Y} \circ \partial$, i.e.

$$
d=\mathbf{Y}_{p+1} \circ \partial: \Omega_{Y}^{p}\left(\mathbb{R}^{D}\right) \rightarrow \Omega_{Y}^{p+1}\left(\mathbb{R}^{D}\right)
$$

where $\mathbf{Y}_{p}$ is now the Young symmetrizer on tensor of degree $p$ associated to the Young symmetry $Y_{p}$. This $d$ is again a first order differential operator which is of degree one, (i.e. it increases the tensorial degree by one), but now, $d^{2} \neq 0$ in general. Instead, one has the following result.

LEMMA 6. Let $N$ be an integer with $N \geq 2$ and assume that $Y$ is such that the number of columns of the Young diagram $Y_{p}$ is strictly smaller than $N$ (i.e. $\leq N-1)$ for any $p \in \mathbb{N}$. Then one has $d^{N}=0$.

In fact the indices in one column are antisymmetrized and $d^{N} \omega$ involves necessarily at least two partial derivatives $\partial$ in one of the columns since there are $N$ partial derivatives involved and at most $N-1$ columns.

Thus if $Y$ satisfies the condition of Lemma $6,\left(\Omega_{Y}\left(\mathbb{R}^{D}\right), d\right)$ is an $N$-complex. Notice that $\Omega_{Y}^{p}\left(\mathbb{R}^{D}\right)=0$ if the first column of $Y_{p}$ contains more than $D$ cells and that therefore, if $Y$ satisfies the condition of Lemma 6 , then $\Omega_{Y}^{p}\left(\mathbb{R}^{D}\right)=0$ for $p>(N-1) D$. 
One can also define a graded bilinear product on $\Omega_{Y}\left(\mathbb{R}^{D}\right)$ by setting

$$
(\alpha \beta)(x)=\mathbf{Y}_{a+b}(\alpha(x) \otimes \beta(x))
$$

for $\alpha \in \Omega_{Y}^{a}\left(\mathbb{R}^{D}\right), \beta \in \Omega_{Y}^{b}\left(\mathbb{R}^{D}\right)$ and $x \in \mathbb{R}^{D}$. This product is by construction bilinear with respect to the $C^{\infty}\left(\mathbb{R}^{D}\right)$-module structure of $\Omega_{Y}\left(\mathbb{R}^{D}\right),\left(\Omega_{Y}^{0}\left(\mathbb{R}^{D}\right)=C^{\infty}\left(\mathbb{R}^{D}\right)\right)$. However it is generically non associative.

In the following we shall not stay at this level of generality but, for each $N \geq 2$ we shall choose a particular $Y$, denoted by $Y^{N}=\left(Y_{p}^{N}\right)_{p \in \mathbb{N}}$, satisfying the condition of Lemma 6 which is maximal in the sense that all the rows are of maximal length $N-1$ except the last one (eventually). In other words the Young diagram with $p$ cells $Y_{p}^{N}$ is defined in the following manner: write the division of $p$ by $N-1$, i.e. write $p=(N-1) n_{p}+r_{p}$ where $n_{p}$ and $r_{p}$ are (the unique) integers with $0 \leq n_{p}$ and $0 \leq r_{p} \leq N-2\left(n_{p}\right.$ is the quotient whereas $r_{p}$ is the remainder $)$, and let $Y_{p}^{N}$ be the Young diagram with $n_{p}$ rows of $N-1$ cells and the last row with $r_{p}$ cells (if $\left.r_{p} \neq 0\right)$. One has $Y_{p}^{N}=\left((N-1)^{n_{p}}, r_{p}\right)$, that is we fill the rows maximally.

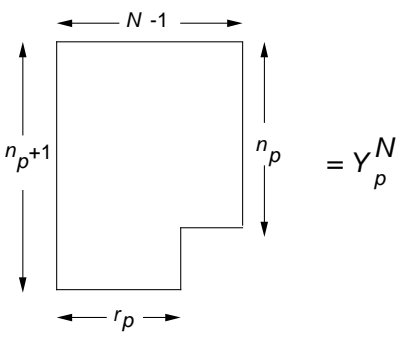

We shall denote $\Omega_{Y^{N}}\left(\mathbb{R}^{D}\right)$ and $\Omega_{Y^{N}}^{p}\left(\mathbb{R}^{D}\right)$ by $\Omega_{N}\left(\mathbb{R}^{D}\right)$ and $\Omega_{N}^{p}\left(\mathbb{R}^{D}\right)$. It is clear that $\left(\Omega_{2}\left(\mathbb{R}^{D}\right), d\right)$ is the usual complex $\left(\Omega\left(\mathbb{R}^{D}\right), d\right)$ of differential forms on $\mathbb{R}^{D}$. The $N$-complex $\left(\Omega_{N}\left(\mathbb{R}^{D}\right), d\right)$ will be simply denoted by $\Omega_{N}\left(\mathbb{R}^{D}\right)$. The Poincaré lemma admits the following generalization [17, 18.

THEOREM 3. One has $H_{(k)}^{(N-1) n}\left(\Omega_{N}\left(\mathbb{R}^{D}\right)\right)=0, \forall n \geq 1$ and $H_{(k)}^{0}\left(\Omega_{N}\left(\mathbb{R}^{D}\right)\right)$ is the space of real polynomial functions on $\mathbb{R}^{D}$ of degree strictly less than $k$ (i.e. $\leq k-1)$ for $k \in\{1, \ldots, N-1\}$.

This statement reduces to the Poincaré lemma for $N=2$ but it is a nontrivial generalization for $N \geq 3$ in the sense that, the spaces $H_{(k)}^{p}\left(\Omega_{N}\left(\mathbb{R}^{D}\right)\right)$ are nontrivial for $p \neq(N-1) n$ and in fact generically infinite dimensional for $D \geq 3, p \geq N$.

The connection between the complex of differential forms on $\mathbb{R}^{D}$ and the theory of classical gauge field of spin 1 is well known. Namely the subcomplex

$$
\Omega^{0}\left(\mathbb{R}^{D}\right) \stackrel{d}{\rightarrow} \Omega^{1}\left(\mathbb{R}^{D}\right) \stackrel{d}{\rightarrow} \Omega^{2}\left(\mathbb{R}^{D}\right) \stackrel{d}{\rightarrow} \Omega^{3}\left(\mathbb{R}^{D}\right)
$$

has the following interpretation in terms of spin 1 gauge field theory. The space $\Omega^{0}\left(\mathbb{R}^{D}\right)\left(=C^{\infty}\left(\mathbb{R}^{D}\right)\right)$ is the space of infinitesimal gauge transformations, the space $\Omega^{1}\left(\mathbb{R}^{D}\right)$ is the space of gauge potentials (which are the appropriate description of spin 1 gauge fields to introduce local interactions). The subspace $d \Omega^{0}\left(\mathbb{R}^{D}\right)$ of $\Omega^{1}\left(\mathbb{R}^{D}\right)$ is the space of pure gauge configurations (which are physically irrelevant), $d \Omega^{1}\left(\mathbb{R}^{D}\right)$ is the space of field strengths or curvatures of gauge potentials. The 
identity $d^{2}=0$ ensures that the curvatures do not see the irrelevant pure gauge potentials whereas, at this level, the Poincaré lemma ensures that it is only these irrelevant configurations which are forgotten when one passes from gauge potentials to curvatures (by applying $d$ ). Finally $d^{2}=0$ also ensures that curvatures of gauge potentials satisfy the Bianchi identity, i.e. are in $\operatorname{Ker}\left(d: \Omega^{2}\left(\mathbb{R}^{D}\right) \rightarrow \Omega^{3}\left(\mathbb{R}^{D}\right)\right)$, whereas at this level the Poincaré lemma implies that conversely the Bianchi identity characterizes the elements of $\Omega^{2}\left(\mathbb{R}^{D}\right)$ which are curvatures of gauge potentials.

Classical spin 2 gauge field theory is the linearization of Einstein geometric theory. In this case, and more generally in the linearization of (pseudo)riemannian geometry, the analog of (11) is a complex $\mathcal{E}^{1} \stackrel{d_{1}}{\rightarrow} \mathcal{E}^{2} \stackrel{d_{2}}{\rightarrow} \mathcal{E}^{3} \stackrel{d_{3}}{\rightarrow} \mathcal{E}^{4}$ where $\mathcal{E}^{1}$ is the space of covariant vector field $\left(x \mapsto X_{\mu}(x)\right)$ on $\mathbb{R}^{D}, \mathcal{E}^{2}$ is the space of covariant symmetric tensor fields of degree $2\left(x \mapsto h_{\mu \nu}(x)\right)$ on $\mathbb{R}^{D}, \mathcal{E}^{3}$ is the space of covariant tensor fields of degree $4\left(x \mapsto R_{\lambda \mu, \rho \nu}(x)\right)$ on $\mathbb{R}^{D}$ having the symmetries of the Riemann curvature tensor and where $\mathcal{E}^{4}$ is the space of covariant tensor fields of degree 5 on $\mathbb{R}^{D}$ having the symmetries of the left-hand side of the Bianchi identity. The arrows $d_{1}, d_{2}, d_{3}$ are given by

$$
\begin{aligned}
& \left(d_{1} X\right)_{\mu \nu}(x)=\partial_{\mu} X_{\nu}(x)+\partial_{\nu} X_{\mu}(x) \\
& \left(d_{2} h\right)_{\lambda \mu, \rho \nu}(x)=\partial_{\lambda} \partial_{\rho} h_{\mu \nu}(x)+\partial_{\mu} \partial_{\nu} h_{\lambda \rho}(x)-\partial_{\mu} \partial_{\rho} h_{\lambda \nu}(x)-\partial_{\lambda} \partial_{\nu} h_{\mu \rho}(x) \\
& \left(d_{3} R\right)_{\lambda \mu \nu, \alpha \beta}(x)=\partial_{\lambda} R_{\mu \nu, \alpha \beta}(x)+\partial_{\mu} R_{\nu \lambda, \alpha \beta}(x)+\partial_{\nu} R_{\lambda \mu, \alpha \beta}(x)
\end{aligned}
$$

The symmetry of $x \mapsto R_{\lambda \mu, \rho \nu}(x),\left(\begin{array}{|l|l|}\hline \lambda & \rho \\ \hline \mu & \nu \\ \hline\end{array}\right)$, shows that $\mathcal{E}^{3}=\Omega_{3}^{4}\left(\mathbb{R}^{D}\right)$ and that $\mathcal{E}^{4}=\Omega_{3}^{5}\left(\mathbb{R}^{D}\right)$; furthermore one canonically has $\mathcal{E}^{1}=\Omega_{3}^{1}\left(\mathbb{R}^{D}\right)$ and $\mathcal{E}^{2}=\Omega_{3}^{2}\left(\mathbb{R}^{D}\right)$. One also sees that $d_{1}$ and $d_{3}$ are proportional to the 3 -differential $d$ of $\Omega_{3}\left(\mathbb{R}^{D}\right)$, i.e. $d_{1} \sim d: \Omega_{3}^{1}\left(\mathbb{R}^{D}\right) \rightarrow \Omega_{3}^{2}\left(\mathbb{R}^{D}\right)$ and $d_{3} \sim d: \Omega_{3}^{4}\left(\mathbb{R}^{D}\right) \rightarrow \Omega_{3}^{5}\left(\mathbb{R}^{D}\right)$. The structure of $d_{2}$ looks different, it is of second order and increases by 2 the tensorial degree. However it is easy to see that it is proportional to $d^{2}: \Omega_{3}^{2}\left(\mathbb{R}^{D}\right) \rightarrow \Omega_{3}^{4}\left(\mathbb{R}^{D}\right)$. Thus the analog of (B) is (for spin 2 gauge field theory)

$$
\Omega_{3}^{1}\left(\mathbb{R}^{D}\right) \stackrel{d}{\rightarrow} \Omega_{3}^{2}\left(\mathbb{R}^{D}\right) \stackrel{d^{2}}{\rightarrow} \Omega_{3}^{4}\left(\mathbb{R}^{D}\right) \stackrel{d}{\rightarrow} \Omega_{3}^{5}\left(\mathbb{R}^{D}\right)
$$

and the fact that it is a complex follows from $d^{3}=0$ whereas the generalized Poincare lemma (Theorem 3) implies that it is in fact an exact sequence. Exactness at $\Omega_{3}^{2}\left(\mathbb{R}^{D}\right)$ is $H_{(2)}^{2}\left(\Omega_{3}\left(\mathbb{R}^{D}\right)\right)=0$ and exactness at $\Omega_{3}^{4}\left(\mathbb{R}^{D}\right)$ is $H_{(1)}^{4}\left(\Omega_{3}\left(\mathbb{R}^{D}\right)\right)=0$, (the exactness at $\Omega_{3}^{4}\left(\mathbb{R}^{D}\right)$ is the main statement of $[33$ ).

Thus what plays the role of the complex of differential forms for the spin 1 (i.e. $\Omega_{2}\left(\mathbb{R}^{D}\right)$ ) is the 3 -complex $\Omega_{3}\left(\mathbb{R}^{D}\right)$ for the spin 2 . More generally, for the spin $S \in \mathbb{N}$, this role is played by the $(S+1)$-complex $\Omega_{S+1}\left(\mathbb{R}^{D}\right)$. In particular, the analog of Sequence (1) for spin 1 is the complex

$$
\Omega_{S+1}^{S-1}\left(\mathbb{R}^{D}\right) \stackrel{d}{\rightarrow} \Omega_{S+1}^{S}\left(\mathbb{R}^{D}\right) \stackrel{d^{S}}{\rightarrow} \Omega_{S+1}^{2 S}\left(\mathbb{R}^{D}\right) \stackrel{d}{\rightarrow} \Omega_{S+1}^{2 S+1}\left(\mathbb{R}^{D}\right)
$$

for the spin $S$. The fact that (3) is a complex was known, [10], here it follows from $d^{S+1}=0$. One easily recognizes that $d^{S}: \Omega_{S+1}^{S}\left(\mathbb{R}^{D}\right) \rightarrow \Omega_{S+1}^{2 S}\left(\mathbb{R}^{D}\right)$ is the generalized (linearized) curvature of 10. Theorem 3 implies that sequence (3) is exact: exactness at $\Omega_{S+1}^{S}\left(\mathbb{R}^{D}\right)$ is $H_{(S)}^{S}\left(\Omega_{S+1}\left(\mathbb{R}^{D}\right)\right)=0$ whereas exactness at $\Omega_{S+1}^{2 S}\left(\mathbb{R}^{D}\right)$ is $H_{(1)}^{2 S}\left(\Omega_{S+1}\left(\mathbb{R}^{D}\right)=0\right.$, (exactness at $\Omega_{S+1}^{S}\left(\mathbb{R}^{D}\right)$ was directly proved in $[9]$ for the 
case $S=3)$.

Finally, there is a generalization of Hodge duality for $\Omega_{N}\left(\mathbb{R}^{D}\right)$, which is obtained by contractions of the columns with the Kroneker tensor $\varepsilon^{\mu_{1} \ldots \mu_{D}}$ of $\mathbb{R}^{D}[\mathbf{1 7}$, 18. When combined with Theorem 3, this duality leads to another kind of results. A typical result of this kind is the following one. Let $T^{\mu \nu}$ be a symmetric contravariant tensor field of degree 2 on $\mathbb{R}^{D}$ satisfying $\partial_{\mu} T^{\mu \nu}=0$, (like e.g. the stress energy tensor), then there is a contravariant tensor field $R^{\lambda \mu \rho \nu}$ of degree 4 with the symmetry \begin{tabular}{|l|l|}
\hline$\lambda$ & $\rho$ \\
\hline$\mu$ & $\nu$ \\
\hline
\end{tabular} , (i.e. the symmetry of Riemann curvature tensor), such that

$$
T^{\mu \nu}=\partial_{\lambda} \partial_{\rho} R^{\lambda \mu \rho \nu}
$$

In order to connect this result with Theorem 3, define $\tau_{\mu_{1} \ldots \mu_{D-1} \nu_{1} \ldots \nu_{D-1}}=$ $T^{\mu \nu} \varepsilon_{\mu \mu_{1} \ldots \mu_{D-1}} \varepsilon_{\nu \nu_{1} \ldots \nu_{D-1}}$. Then one has $\tau \in \Omega_{3}^{2(D-1)}\left(\mathbb{R}^{D}\right)$ and conversely, any $\tau \in \Omega_{3}^{2(D-1)}\left(\mathbb{R}^{D}\right)$ can be expressed in this form in terms of a symmetric contravariant 2-tensor. It is easy to verify that $d \tau=0$ (in $\Omega_{3}\left(\mathbb{R}^{D}\right)$ ) is equivalent to $\partial_{\mu} T^{\mu \nu}=0$. On the other hand, Theorem 3 implies that $H_{(1)}^{2(D-1)}\left(\Omega_{3}\left(\mathbb{R}^{D}\right)\right)=0$ and therefore $\partial_{\mu} T^{\mu \nu}=0$ implies that there is a $\rho \in \Omega_{3}^{2(D-2)}\left(\mathbb{R}^{D}\right)$ such that $\tau=d^{2} \rho$. The latter is equivalent to the above equation with $R^{\mu_{1} \mu_{2} \nu_{1} \nu_{2}}$ proportional to $\varepsilon^{\mu_{1} \mu_{2} \ldots \mu_{D}} \varepsilon^{\nu_{1} \nu_{2} \ldots \nu_{D}} \rho_{\mu_{3} \ldots \mu_{D} \nu_{3} \ldots \nu_{D}}$ and one verifies that, so defined, $R$ has the correct symmetry. This result has been used in [65] in the investigation of the consistent deformations of the free spin two gauge field action.

\section{Graded differential algebras and generalizations}

A graded differential algebra is a (cochain) complex $\mathfrak{A}=\oplus_{n \in \mathbb{Z}} \mathfrak{A}^{n}$ with differential $d$ such that $\mathfrak{A}$ is a $\mathbb{Z}$-graded associative unital $\mathbf{k}$-algebra and such that $d$ is an antiderivation i.e. satisfies the graded Leibniz rule

$$
d(\alpha \beta)=d(\alpha) \beta+(-1)^{a} \alpha d(\beta)
$$

for any $\alpha \in \mathfrak{A}^{a}, \beta \in \mathfrak{A}$ and where $(\alpha, \beta) \mapsto \alpha \beta$ denotes the product of $\mathfrak{A}$. If $\mathfrak{A}$ is such a graded differential algebra with differential $d, \operatorname{Ker}(d)$ is a graded unital subalgebra of $\mathfrak{A}$ whereas $\operatorname{Im}(d)$ is a graded two-sided ideal of $\operatorname{Ker}(d)$ so the cohomology $H(\mathfrak{A})$ is a (unital associative) graded algebra. If $\mathfrak{A}$ and $\mathfrak{B}$ are two graded differential algebras, the tensor product $\mathfrak{A} \otimes \mathfrak{B}$ of the underlying complexes (as defined in Section 3) is again a graded differential algebra with product defined by

$$
(\alpha \otimes \beta)\left(\alpha^{\prime} \otimes \beta^{\prime}\right)=(-1)^{b a^{\prime}} \alpha \alpha^{\prime} \otimes \beta \beta^{\prime}
$$

for $\alpha \in \mathfrak{A}, \beta \in \mathfrak{B}^{b}, \alpha^{\prime} \in \mathfrak{A}^{a^{\prime}}$ and $\beta^{\prime} \in \mathfrak{B}$. In the following, the product of a tensor product of graded algebras will be always the above one. With this convention, if $\mathbf{k}$ is a field one has $H(\mathfrak{A} \otimes \mathfrak{B})=H(\mathfrak{A}) \otimes H(\mathfrak{B})$ for the corresponding cohomology algebras (which is the refined counterpart of Proposition 2 for graded differential algebras).

Let $\left(\mathfrak{A}^{n}\right)_{n \in \mathbb{N}}$ be a pre-cosimplicial module (see in Section 3) such that $\mathfrak{A}=\oplus \mathfrak{A}^{n}$ is a (positively) graded algebra and assume that the cofaces homomorphisms $\mathfrak{f}_{i}$ satisfy the following assumptions $(\mathfrak{M F})$ : 


$$
\mathfrak{f}_{i}(\alpha \beta)=\left\{\begin{array}{ll}
\mathfrak{f}_{i}(\alpha) \beta & \text { if } \quad i \leq a \\
\alpha \mathfrak{f}_{i-a}(\beta) & \text { if } \quad i>a
\end{array}, i \in\{0, \ldots, a+b+1\}\right.
$$

and

$\left(\mathfrak{M F}_{2}\right) \quad \mathfrak{f}_{a+1}(\alpha) \beta=\alpha \mathfrak{f}_{0}(\beta)$

for $\alpha \in \mathfrak{A}^{a}$ and $\beta \in \mathfrak{A}^{b}$ (where $(\alpha, \beta) \mapsto \alpha \beta$ denote the product of $\mathfrak{A}$ ). Then the corresponding complex $(\mathfrak{A}, d)$ is a graded differential algebra. If furthermore $\left(\mathfrak{A}^{n}\right)$ is a cosimplicial module with codegeneracy homomorphisms $\mathfrak{s}_{i}$ satisfying the following assumption (MS)

$$
(\mathfrak{M S}) \quad \mathfrak{s}_{i}(\alpha \beta)= \begin{cases}\mathfrak{s}_{i}(\alpha) \beta & \text { if } \quad i<a \\ \alpha \mathfrak{s}_{i-a}(\beta) & \text { if } \quad i \geq a\end{cases}
$$

$i \in\{0, \ldots, a+b-1\}$, then the subcomplex $N(\mathfrak{A})$ of normalized cochains of $\mathfrak{A}$ is a graded differential subalgebra of $\mathfrak{A}$. In [14], a pre-cosimplicial module $\left(\mathfrak{A}^{n}\right)$ as above with cofaces satisfying $(\mathfrak{M F})$ (which was denoted there by $(\mathfrak{A F})$ ) was called a pre-cosimplicial algebra and in the case where $\left(\mathfrak{A}^{n}\right)$ is furthermore a cosimplicial

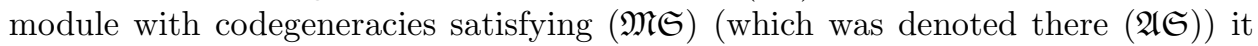
was called a cosimplicial algebra, however it has been remarked by Max Karoubi that this terminology is misleading so we shall speak in the following of a $\mathfrak{M}$-precosimplicial module in the first case and of a $\mathfrak{M}$-cosimplicial module in the second case. In fact $\mathfrak{M}$-cosimplicial modules is what corresponds to graded differential algebras in an appropriate specific version of the Dold-Kan correspondence.

Let $\mathcal{A}$ be an associative unital $\mathbf{k}$-algebra and $\mathcal{M}$ be a $(\mathcal{A}, \mathcal{A})$-bimodule. As pointed out in Section 3, the $\mathcal{M}$-valued Hochschild cochains give rise to a cosimplicial module $\left(C^{n}(\mathcal{A}, \mathcal{M})\right)_{n \in \mathbb{N}}$. In the case $\mathcal{M}=\mathcal{A}, C(\mathcal{A}, \mathcal{A})$ has a natural structure of $\mathbb{N}$-graded associative unital $\mathbf{k}$-algebra with product $(\alpha, \beta) \mapsto \alpha \beta$ given by

$$
\alpha \beta\left(x_{1}, \ldots, x_{a+b}\right)=\alpha\left(x_{1}, \ldots, x_{a}\right) \beta\left(x_{a+1}, \ldots, x_{a+b}\right),
$$

for $\alpha \in C^{a}(\mathcal{A}, \mathcal{A}), \beta \in C^{b}(\mathcal{A}, \mathcal{A}), x_{i} \in \mathcal{A}$.

It is easily verified that the assumptions (MF) and (MS) are satisfied so that $\left(C^{n}(\mathcal{A}, \mathcal{A})\right)$ is a $\mathfrak{M}$-cosimplicial module. Thus $C(\mathcal{A}, \mathcal{A})$ equipped with the simplicial (Hochschild) differential (as in Section 3) is a graded differential algebra and the submodule of normalized cochains is a graded differential subalgebra of $C(\mathcal{A}, \mathcal{A})$.

Let again $\mathcal{A}$ be an associative unital k-algebra and let us denote by $\mathfrak{T}(\mathcal{A})=$ $\oplus_{n \in \mathbb{N}} \mathfrak{T}^{n}(\mathcal{A})$ the tensor algebra over $\mathcal{A}$ of the $(\mathcal{A}, \mathcal{A})$-bimodule $\mathcal{A} \otimes \mathcal{A}$. This is a (positively) graded associative unital k-algebra with $\mathfrak{T}^{n}(\mathcal{A})=\otimes^{n+1} \mathcal{A}$ and product $\left(x_{0} \otimes \cdots \otimes x_{n}\right)\left(y_{0} \otimes \cdots \otimes y_{m}\right)=x_{0} \otimes \cdots \otimes x_{n-1} \otimes x_{n} y_{0} \otimes y_{1} \otimes \cdots \otimes y_{m}$ for $x_{i}, y_{j} \in \mathcal{A}$. One verifies that one defines a structure of $\mathfrak{M}$-cosimplicial module for $\left(\mathfrak{T}^{n}(\mathcal{A})\right)$ by setting

$\mathfrak{f}_{0}\left(x_{0} \otimes \cdots \otimes x_{n}\right)=\mathbb{1} \otimes x_{0} \otimes \cdots \otimes x_{n}$

$\mathfrak{f}_{i}\left(x_{0} \otimes \cdots \otimes x_{n}\right)=x_{0} \otimes \cdots \otimes x_{i-1} \otimes \mathbb{1} \otimes x_{i} \otimes \cdots \otimes x_{n}$ for $1 \leq i \leq n$

$\mathfrak{f}_{n+1}\left(x_{0} \otimes \cdots \otimes x_{n}\right)=x_{0} \otimes \cdots \otimes x_{n} \otimes \mathbb{1}$

and

$\mathfrak{s}_{i}\left(x_{0} \otimes \cdots \otimes x_{n}\right)=x_{0} \otimes \cdots \otimes x_{i} x_{i+1} \otimes \cdots \otimes x_{n}$ for $0 \leq i \leq n-1$.

It follows that, equipped with the corresponding simplicial differential, $\mathfrak{T}(\mathcal{A})$ is a graded differential algebra and that the submodule of normalized cochains is a graded differential subalgebra of $\mathfrak{T}(\mathcal{A})$. This latter graded differential algebra will be denoted by $\Omega(\mathcal{A})$ and referred to as the universal graded differential envelope 
of $\mathcal{A}$ or simply the universal differential envelope of $\mathcal{A}$; it is characterized by the following universal property [41], 42] (see also e.g. in [8] and [16]).

PROPOSITION 5. Any homomorphism $\varphi$ of unital algebras of $\mathcal{A}$ into the subalgebra $\Omega^{0}$ of elements of degree 0 of a graded differential algebra $\Omega$ has a unique extension $\tilde{\varphi}: \Omega(\mathcal{A}) \rightarrow \Omega$ as a homomorphism of graded differential algebras.

The graded differential algebra $\Omega(\mathcal{A})$ is usually constructed in a different manner; the fact that it identifies with the graded differential algebra of normalized cochains of $\mathfrak{T}(\mathcal{A})$ is well known. It is worth noticing here that $\Omega(\mathcal{A})$ is also the graded differential subalgebra of $\mathfrak{T}(\mathcal{A})$ generated by $\mathcal{A}$ (i.e. the smallest graded differential subalgebra which contains $\mathcal{A}$ ).

We now come to an $N$-complex version of graded differential algebra $(N \geq 2)$. For that we shall need $q \in \mathbf{k}$ such that Assumption $\left(A_{1}\right)$ of Section 5 is satisfied i.e. $[N]_{q}=0$ and $[n]_{q}$ invertible in $\mathbf{k}$ for $n \in\{1, \cdots, N-1\}$. Throughout the following of this section, $N$ and $q \in \mathbf{k}$ are fixed and such that $\left(A_{1}\right)$ is satisfied. The following lemma is basic for the generalization, 14. In this lemma, (and in the following) $d_{1}$ is the $N$-differential defined in Section 6 for any pre-cosimplicial module.

LEMMA 7. Suppose that $\mathbf{k}$ and $q \in \mathbf{k}$ satisfy Assumption $\left(A_{1}\right)$ and let $\left(\mathfrak{A}^{n}\right)$ be a M-pre-cosimplicial module. Then the $N$-differential $d_{1}$ satisfies the graded q-Leibniz rule, that is

$$
d_{1}(\alpha \beta)=d_{1}(\alpha) \beta+q^{a} \alpha d_{1}(\beta)
$$

for $\alpha \in \mathfrak{A}^{a}$ and $\beta \in \mathfrak{A}=\oplus_{n} \mathfrak{A}^{n}$.

A unital associative graded algebra equipped with an $N$-differential satisfying the (above) graded $q$-Leibniz rule will be referred to as a graded $q$-differential algebra [20], 14]. The content of the above lemma is that if $\left(\mathfrak{A}^{n}\right)$ is a $\mathfrak{M}$-pre-cosimplicial module then $\left(\mathfrak{A}, d_{1}\right)$ is a graded $q$-differential algebra which is positively graded. If, furthermore $\left(\mathfrak{A}^{n}\right)$ is a $\mathfrak{M}$-cosimplicial module then the generalized cohomology of $\left(\mathfrak{A}, d_{1}\right)$ is given in terms of the ordinary cohomology of $\left(\mathfrak{A}^{n}\right)$ by Theorem 2 .

Let $\mathcal{A}$ be as above an associative unital algebra. It follows from Lemma 7 that $\mathfrak{T}(\mathcal{A})$ equipped with the $N$-differential $d_{1}$ is a graded $q$-differential algebra (which is $\mathbb{N}$-graded). Let $\Omega_{q}(\mathcal{A})$ be the graded $q$-differential subalgebra of $\mathfrak{T}(\mathcal{A})$ generated by $\mathcal{A}$, i.e. the smallest subalgebra of $\mathfrak{T}(\mathcal{A})$ which contains $\mathcal{A}$ and which is stable by the $N$-differential $d_{1}$. As graded $q$-differential algebra, $\Omega_{q}(\mathcal{A})$ is characterized uniquely up to an isomorphism by the following universal property [20], 14].

PROPOSITION 6. Any homomorphism $\varphi$ of unital algebras of $\mathcal{A}$ into the subalgebra $\Omega^{0}$ of elements of degree 0 of a graded q-differential algebra $\Omega$ has a unique extension $\tilde{\varphi}: \Omega_{q}(\mathcal{A}) \rightarrow \Omega$ as a homomorphism of graded q-differential algebras.

This is the $q$-analog of Proposition 5, (a homomorphism of graded $q$-differential algebra being a homomorphism of graded algebras permuting the $N$-differentials). For $N=2, \Omega_{q}(\mathcal{A})$ reduces to $\Omega(\mathcal{A})$. The graded $q$-differential algebra $\Omega_{q}(\mathcal{A})$ is referred to as the universal $q$-differential envelope of $\mathcal{A}$ [20], [14]. The generalized cohomologies of $\left(\mathfrak{T}(\mathcal{A}), d_{1}\right)$ and of $\Omega_{q}(\mathcal{A})$ are generically trivial; one has the following result, [14]. 
LECTURES ON DIFFERENTIALS, GENERALIZED DIFFERENTIALS AND...

PROPOSITION 7. Assume that $\mathcal{A}$ admits a linear form $\omega \in \mathcal{A}^{*}$ such that $\omega(\mathbb{1})=1$. Then the generalized cohomologies of $\left(\mathfrak{T}(\mathcal{A}), d_{1}\right)$ and of $\Omega_{q}(\mathcal{A})$ are given by:

$$
\begin{aligned}
& H_{(k)}^{n}\left(\mathfrak{T}(\mathcal{A}), d_{1}\right)=H_{(k)}^{n}\left(\Omega_{q}(\mathcal{A})\right)=0 \quad \text { for } n \geq 1 \text { and } \\
& H_{(k)}^{0}\left(\mathfrak{T}(\mathcal{A}), d_{1}\right)=H_{(k)}^{0}\left(\Omega_{q}(\mathcal{A})\right)=\mathbf{k}, \quad \forall k \in\{1, \cdots, N-1\} .
\end{aligned}
$$

Notice that the assumption of this proposition is satisfied if $\mathbf{k}$ is a field and that the case $N=2$ means, under the same assumption, the triviality of the cohomologies of $\mathfrak{T}(\mathcal{A})$ and $\Omega(\mathcal{A})$, (a well known fact, 42 ).

The above discussion shows the naturality of the notion of graded $q$-differential algebra as " $N$-generalization" or $q$-analog of the notion of graded differential algebra. This notion has a slight drawback which is the non existence of natural tensor products [55]; let us discuss this point. It was shown in [40] that if $q \in \mathbf{k}$ is such that Assumption $\left(A_{1}\right)$ is satisfied then one can construct a tensor product for $N$-complexes in the following manner. Let $\left(E^{\prime}, d^{\prime}\right)$ and $\left(E^{\prime \prime}, d^{\prime \prime}\right)$ be two $N$-complexes and let us define $d$ on $E^{\prime} \otimes E^{\prime \prime}$ by setting

$$
d\left(\alpha^{\prime} \otimes \alpha^{\prime \prime}\right)=d^{\prime}\left(\alpha^{\prime}\right) \otimes \alpha^{\prime \prime}+q^{a^{\prime}} \alpha^{\prime} \otimes d^{\prime \prime}\left(\alpha^{\prime \prime}\right), \forall \alpha^{\prime} \in E^{\prime a^{\prime}}, \forall \alpha^{\prime \prime} \in E^{\prime \prime},
$$

one has by induction on $n \in \mathbb{N}$

$$
d^{n}\left(\alpha^{\prime} \otimes \alpha^{\prime \prime}\right)=\sum_{m=0}^{n} q^{a^{\prime}(n-m)}\left[\begin{array}{l}
n \\
m
\end{array}\right]_{q} d^{\prime m}\left(\alpha^{\prime}\right) \otimes d^{\prime \prime n-m}\left(\alpha^{\prime \prime}\right),
$$

therefore Assumption $\left(A_{1}\right)$ implies $d^{N}\left(\alpha^{\prime} \otimes \alpha^{\prime \prime}\right)=d^{\prime N}\left(\alpha^{\prime}\right) \otimes \alpha^{\prime \prime}+\alpha^{\prime} \otimes d^{\prime \prime N}\left(\alpha^{\prime \prime}\right)=0$. Unfortunately, as pointed out in [55], when $\left(E^{\prime}, d^{\prime}\right)$ and $\left(E^{\prime \prime}, d^{\prime \prime}\right)$ are furthermore two graded $q$-differential algebras, $d$ fails to be a $q$-differential in that it does not satisfy the graded $q$-Leibniz rule except for $q=1$ or $q=-1$.

As for $N$-complexes, many notions for graded $q$-differential algebras do only depend on the underlying $\mathbb{Z}_{N^{-}}$graduation so it is natural to consider the following $\mathbb{Z}_{N^{-}}$ graded version. $\mathrm{A} \mathbb{Z}_{N}$-graded $q$-differential algebra is a $\mathbb{Z}_{N}$-graded algebra equipped with a homogeneous endomorphism $d$ of degree 1 which is an $N$-differential, i.e. $d^{N}=0$, and which satisfies the graded $q$-Leibniz rule $d(\alpha \beta)=d(\alpha) \beta+q^{a} \alpha d(\beta)$ for $\alpha$ homogeneous of degree $a \in \mathbb{Z}_{N}$, (let us remind that $N$ and $q \in \mathbf{k}$ are connected by Assumption $\left(A_{1}\right)$ ). We have already met such a $\mathbb{Z}_{N}$-graded $q$-differential algebra at the end of Section 6 , (namely $M_{N}(\mathbf{k})$ ).

The notion of graded $q$-differential algebra was introduced in 20$]$ for $\mathbf{k}=\mathbb{C}$ as well as the construction of the universal $q$-differential envelopes. Here, we have followed the presentation of 14 .

\section{Subquotients and constraints}

Let $E$ be a module and let $u \in E^{*}$ be a linear form on $E$. To these data, one associates a graded differential algebra $K(u)$ which is constructed in the following manner. As an algebra, $K(u)$ is the exterior algebra (over $\mathbf{k}) \wedge E$ of $E$ but it is equipped with the opposite graduation, i.e. $K(u)=\oplus_{n} K^{n}(u)$ with $K^{n}(u)=\wedge^{-n} E$ if $n \leq 0$ and $K^{n}(u)=0$ if $n>0$. The differential $d_{u}$ of $K(u)$ is then defined to be the unique homogeneous k-linear endomorphism of degree 1 of $K(u)$ satisfying the graded Leibniz rule and such that $d_{u}(e)=u(e) \in \mathbf{k}=K^{0}(u)$ for any 
$e \in E=K^{-1}(u)$. One has $d_{u}^{2}=0$ so $K(u)$ is a graded differential algebra; the underlying complex is a Koszul complex and will be referred to as the Koszul complex associated to the pair $(E, u)$.

Let $M$ be a smooth (finite-dimensional, connected, paracompact) manifold and let $V$ be a closed submanifold of $M$. The $\mathbb{R}$-algebra $C^{\infty}(M)$ of smooth functions on $M$ will play the role of $\mathbf{k}$ and $I(V)$ will denote the ideal of a smooth function on $M$ vanishing on $V$. We introduce the following regularity assumption $\left(R_{0}\right)$ for the data $(M, V)$ :

$\left(R_{0}\right) I(V)$ is generated by $m$ functions $u_{\alpha} \in C^{\infty}(M), \alpha \in\{1, \cdots, m\}$, which are independent on $V$ in the sense $d u_{1}(x) \wedge \cdots \wedge d u_{m}(x) \neq 0, \forall x \in V$.

Let $E=\mathbb{R}^{m} \otimes_{\mathbb{R}} C^{\infty}(M)$ be the free $C^{\infty}(M)$-module of rank $m$ with canonical basis denoted by $\pi_{\alpha}, \alpha \in\{1, \cdots, m\}$, and let $u \in E^{*}$ be the $\left(C^{\infty}(M)\right.$-)linear form on $E$ defined by $u\left(\pi_{\alpha}\right)=u_{\alpha} \in C^{\infty}(M)$, for $\alpha \in\{1, \cdots, m\}$. The Koszul complex $K(u)$ associated to the pair $(E, u)$ identifies with the free graded-commutative unital $C^{\infty}(M)$-algebra generated by the $\pi_{\alpha}$ in degree -1 equipped with the differential $d_{u}$ above; This is a graded differential $C^{\infty}(M)$-algebra. Under Assumption $\left(R_{0}\right)$ one has with these notations the following result [12].

LEMMA 8. The cohomology $H(K(u))$ of $K(u)$ is given by $H^{n}(K(u))=0$ if $n \neq 0$ and $H^{0}(K(u))$ identifies canonically with the algebra $C^{\infty}(V)$ of smooth functions on $V$.

In fact, $d_{u}\left(K^{-1}(u)\right)=I(V)$ so one has $H^{0}(K(u))=C^{\infty}(M) / I(V)$ which is canonically $C^{\infty}(V)$; notice that $C^{\infty}(V)$ is a $C^{\infty}(M)$-algebra. Notice also that, since the $\mathbb{R}$-algebra $C^{\infty}(M)$ is unital, $K(u)$ is also a graded differential algebra over $\mathbb{R}$.

Lemma 8 gives a homological description of the algebra of functions on a submanifold (under assumption $\left(R_{0}\right)$ ). Our aim is now to give a homological description of the algebra of functions on a quotient manifold and finally to mix both descriptions to obtain a homological description of the algebra of functions on a quotient of a submanifold (subquotient).

Let $V$ be a smooth manifold. Recall that a foliation of $V$ is a vector subbundle $F$ of the tangent bundle $T(V)$ of $V$ which is such that the $C^{\infty}(V)$-module $\mathcal{F}$ of sections of $F$ is also a Lie subalgebra of the Lie algebra of vector fields on $V$. In the following, we shall identify the foliation with $\mathcal{F}$. The ideal $(\wedge \mathcal{F})^{\perp}$ of the algebra $\Omega(V)$ of differential forms on $V$ of forms vanishing on $\mathcal{F}$ is a differential ideal so the quotient $\Omega(V) /(\wedge \mathcal{F})^{\perp}$ is a graded differential algebra over $\mathbb{R}$ which will be denoted by $\Omega(V, \mathcal{F})$ and referred to as the graded differential algebra of longitudinal forms of $\mathcal{F}$; its differential will be denoted by $d_{\mathcal{F}}$. In fact $\Omega(V, \mathcal{F})$ is a subcomplex of the Chevalley-Eilenberg complex $C_{\wedge}\left(\mathcal{F}, C^{\infty}(V)\right.$ ), (see Appendix B), where $\mathcal{F}$ acts by derivations on $C^{\infty}(V)$. Let $H(V, \mathcal{F})$ be the cohomology of longitudinal forms; in degree $0, H^{0}(V, \mathcal{F})$ identifies with the $\mathbb{R}$-algebra of smooth functions on $V$ invariant by the action of the vector fields belonging to $\mathcal{F}$. In the case where the quotient $V / \mathcal{F}$ exists as smooth manifold and is such that the canonical projection $p: V \rightarrow V / \mathcal{F}$ is a submersion, $H^{0}(V, \mathcal{F})$ identifies with the algebra $C^{\infty}(V / \mathcal{F})$ of smooth functions on the quotient. Let us introduce the following regularity assumption $\left(R_{1}\right)$ for 
LECTURES ON DIFFERENTIALS, GENERALIZED DIFFERENTIALS AND...

$(V, \mathcal{F}):$

$\mathcal{F}$ is a free $C^{\infty}(V)$-module of rank $m^{\prime}$, or equivalently
$F$ is a trivial vector bundle of rank $m^{\prime}$.

If $\left(R_{1}\right)$ is satisfied $\Omega(V, \mathcal{F})$ identifies with the graded-commutative algebra $C^{\infty}(V) \otimes_{\mathbb{R}} \wedge \mathbb{R}^{m^{\prime}} ;$ in fact, $\Omega(V, \mathcal{F})$ is then the free graded-commutative unital $C^{\infty}(V)$-algebra generated by the $\chi^{\alpha^{\prime}}$ in degree $1, \alpha^{\prime} \in\left\{1, \cdots, m^{\prime}\right\}$, where $\left(\chi^{\alpha^{\prime}}\right)$ is the dual basis of the basis $\left(\xi_{\alpha^{\prime}}\right)$ of $\mathcal{F}$. With these conventions, $d_{\mathcal{F}}$ is given on the generators by

$$
\left\{\begin{array}{l}
d_{\mathcal{F}} f=\xi_{\alpha^{\prime}}(f) \chi^{\alpha^{\prime}}, \forall f \in C^{\infty}(V) \\
d_{\mathcal{F}} \chi^{\alpha^{\prime}}=-\frac{1}{2} C_{\beta^{\prime} \gamma^{\prime}}^{\alpha^{\prime}} \chi^{\beta^{\prime}} \chi^{\gamma^{\prime}}
\end{array}\right.
$$

the $C_{\beta^{\prime} \gamma^{\prime}}^{\alpha^{\prime}} \in C^{\infty}(V)$ being given by $\left[\xi_{\beta^{\prime}}, \xi_{\gamma^{\prime}}\right]=C_{\beta^{\prime} \gamma^{\prime}}^{\alpha^{\prime}} \xi_{\alpha^{\prime}}$ i.e. $C_{\beta^{\prime} \gamma^{\prime}}^{\alpha^{\prime}}=\chi^{\alpha^{\prime}}\left(\left[\xi_{\beta^{\prime}}, \xi_{\gamma^{\prime}}\right]\right)$. One must be aware of the fact that $\Omega(V, \mathcal{F})$ is a graded differential algebra over $\mathbb{R}$ (and not over $C^{\infty}(V)$ ).

It is worth noticing here that an infinite dimensional analog of the above appears in gauge theory; there, $V$ is replaced by the affine space of gauge potentials (connections), $\mathcal{F}$ is replaced by the Lie algebra of the group of gauge transformations acting on gauge potentials whereas the analog of the $\chi^{\alpha^{\prime}}$ are components of the ghost field $\chi(x)$. In this context the BRS differential [2] corresponds to the longitudinal differential $d_{\mathcal{F}},[\mathbf{6 4}],[\mathbf{5 9}]$.

Let now $M$ be a smooth manifold, $V$ be a closed submanifold of $M$ and assume that $V$ is equipped with a foliation $\mathcal{F}$. We want to combine the above constructions to produce a homological description of $C^{\infty}(V / \mathcal{F})$. More precisely, our aim is to produce a graded differential algebra which contains $C^{\infty}(M)$ and which has the longitudinal cohomology $H(V, \mathcal{F})$ as cohomology. We assume in the following that the assumption $\left(R_{0}\right)$ is satisfied by $(M, V)$ and that the assumption $\left(R_{1}\right)$ is satisfied by $(V, \mathcal{F})$. With $(M, V)$ satisfying $\left(R_{0}\right)$ is associated as above the Koszul complex $K(u)$ with differential $d_{u}$. Let $\mathcal{K}=\oplus_{i, j} \mathcal{K}^{i, j}$ be the bigraded algebra $K(u) \otimes_{\mathbb{R}} \wedge \mathbb{R}^{m^{\prime}}$ with $\mathcal{K}^{i, j}=K^{i}(u) \otimes_{\mathbb{R}} \wedge^{j} \mathbb{R}^{m^{\prime}}$ i.e. $\mathcal{K}^{i, j}=\wedge^{-i} \mathbb{R}^{m} \otimes_{\mathbb{R}} C^{\infty}(M) \otimes_{\mathbb{R}} \wedge^{j} \mathbb{R}^{m^{\prime}}$ if $i \leq 0 \leq j$ and $\mathcal{K}^{i, j}=0$ otherwise. One can also consider that $\mathcal{K}$ is a $\mathbb{Z}$-graded algebra, $\mathcal{K}=\oplus_{n} \mathcal{K}^{n}$, for the total degree $\mathcal{K}^{n}=\oplus_{i+j=n} \mathcal{K}^{i, j}$. We shall again denote by $\pi_{\alpha}, \alpha \in\{1, \cdots, m\}$, and $\chi^{\alpha^{\prime}}, \alpha^{\prime} \in\left\{1, \cdots, m^{\prime}\right\}$ the elements of $\mathcal{K}$ corresponding to the canonical basis of $\mathbb{R}^{m}$ and of $\mathbb{R}^{m^{\prime}}$. As graded $C^{\infty}(M)$-algebra, $\mathcal{K}$ is the free graded-commutative unital $C^{\infty}(M)$-algebra generated by the $\pi_{\alpha}$ in degree -1 and the $\chi^{\alpha^{\prime}}$ in degree 1 . One recovers the bidegree by giving the bidegree $(-1,0)$ to the $\pi_{\alpha}$ and the bidegree $(0,1)$ to the $\chi^{\alpha^{\prime}}$. Let us extend the differential $d_{u}$ of $K(u)$ as the unique antiderivation $\delta_{0}$ of $\mathcal{K}$ such that $\delta_{0} \chi^{\alpha^{\prime}}=0, \delta_{0} f=0$ for $f \in C^{\infty}(M)$ and $\delta_{0} \pi_{\alpha}=u_{\alpha}$; one still has $\delta_{0}^{2}=0$ so $\mathcal{K}$ equipped with $\delta_{0}$ is a graded differential algebra. Furthermore since $\delta_{0}$ is homogeneous for the bidegree (of bidegree $(1,0)$ ) the cohomology $H\left(\delta_{0}\right)$ of $\left(\mathcal{K}, \delta_{0}\right)$ is bigraded, $H\left(\delta_{0}\right)=\oplus_{i, j} H^{i, j}\left(\delta_{0}\right)$, and Lemma 8 implies that $H^{i, j}\left(\delta_{0}\right)=0$ if $i \neq 0$ and that $H^{0, j}\left(\delta_{0}\right)=C^{\infty}(V) \otimes_{\mathbb{R}} \wedge^{j} \mathbb{R}^{m^{\prime}}$ in other words one has the following lemma.

LEMMA 9. As a graded $\mathbb{R}$-algebra, the cohomology $H\left(\delta_{0}\right)$ of $\left(\mathcal{K}, \delta_{0}\right)$ identifies with the graded algebra $\Omega(V, \mathcal{F})$ of longitudinal forms.

The following lemma states that there is an antiderivation of $\mathcal{K}$ which induces the longitudinal differential $d_{\mathcal{F}}$ on $H\left(\delta_{0}\right)$. 
LEMMA 10. There is an antiderivation of $\delta_{1}$ of degree 1 of $\mathcal{K}$ which is homogeneous for the bidegree of bidegree $(0,1)$, which satisfies $\delta_{0} \delta_{1}+\delta_{1} \delta_{0}=0$ and which induces the longitudinal differential $d_{\mathcal{F}}$ on $H\left(\delta_{0}\right)=\Omega(V, \mathcal{F})$.

Proof. The longitudinal differential is given by (4) on $C(V) \otimes_{\mathbb{R}} \wedge \mathbb{R}^{m^{\prime}}$. It follows from our assumptions that there are vector fields $\tilde{\xi}_{\alpha^{\prime}}$ on $M$ such that their restrictions to $V$ are tangent to $V$ and coincide with the $\xi_{\alpha^{\prime}}, \tilde{\xi}_{\alpha^{\prime}} \uparrow V=\xi_{\alpha^{\prime}}$ for $\alpha^{\prime} \in\left\{1, \cdots, m^{\prime}\right\}$. Similarily there are $\tilde{C}_{\beta^{\prime} \gamma^{\prime}}^{\alpha^{\prime}} \in C^{\infty}(M)$ such that $\tilde{C}_{\beta^{\prime} \gamma^{\prime}}^{\alpha^{\prime}} \uparrow V=$ $C_{\beta^{\prime} \gamma^{\prime}}^{\alpha^{\prime}} \in C^{\infty}(V)$. Define then $\delta_{1}$ on $C^{\infty}(M) \otimes_{\mathbb{R}} \wedge \mathbb{R}^{m^{\prime}}$ by

$$
\left\{\begin{array}{l}
\delta_{1} f=\tilde{\xi}_{\alpha^{\prime}}(f) \chi^{\alpha^{\prime}}, \quad \forall f \in C^{\infty}(M) \\
\delta_{1} \chi^{\alpha^{\prime}}=-\frac{1}{2} \tilde{C}_{\beta^{\prime} \gamma^{\prime}}^{\alpha^{\prime}} \chi^{\beta^{\prime}} \chi^{\gamma^{\prime}}
\end{array}\right.
$$

One has $\left(\delta_{0} \delta_{1}+\delta_{1} \delta_{0}\right) f=\delta_{0} \delta_{1} f=0$ and $\left(\delta_{0} \delta_{1}+\delta_{1} \delta_{0}\right) \chi^{\alpha^{\prime}}=\delta_{0} \delta_{1} \chi^{\alpha^{\prime}}=0$ for $f \in C^{\infty}(M)$ and $\alpha^{\prime} \in\left\{1, \cdots, m^{\prime}\right\}$. On the other hand, one has $\delta_{1} \delta_{0} \pi_{\alpha}=\delta_{1} u_{\alpha}=$ $\tilde{\xi}_{\alpha^{\prime}}\left(u_{\alpha}\right) \chi^{\alpha^{\prime}}$ and, by construction $\tilde{\xi}_{\alpha^{\prime}}\left(u_{\alpha}\right)$ vanishes on $V$ so $\tilde{\xi}_{\alpha^{\prime}}\left(u_{\alpha}\right)=A_{\alpha^{\prime} \alpha}^{\beta} u_{\beta}$ for some $A_{\alpha^{\prime} \alpha}^{\beta} \in C^{\infty}(M)$. By setting $\delta_{1} \pi_{\alpha}=-A_{\alpha^{\prime} \alpha}^{\beta} \pi_{\beta} \chi^{\alpha^{\prime}}$ and by extending $\delta_{1}$ to $\mathcal{K}$ by the antiderivation property, one has $\delta_{0} \delta_{1}+\delta_{1} \delta_{0}=0$ so $\delta_{1}$ induces an antiderivation of degree 1 of $H\left(\delta_{0}\right)=C(V) \otimes_{\mathbb{R}} \wedge \mathbb{R}^{m^{\prime}}$ which coincides with $d_{\mathcal{F}}$ in view of (4).

As it is apparent in Formula (5), $\delta_{1}$ is an antiderivation of $\mathcal{K}$ considered as a graded algebra over $\mathbb{R}$ (and not over $C^{\infty}(M)$ in contrast with $\delta_{0}$ ).

LEMMA 11. There are antiderivations $\delta_{r}$ of degree 1 of the graded $\mathbb{R}$-algebra $\mathcal{K}$ with $\delta_{r}$ homogeneous for the bidegree of bidegree $(1-r, r)$ for $r \geq 2$, such that one has with $\delta_{0}$ and $\delta_{1}$ as above $\sum_{r+s=n} \delta_{r} \delta_{s}=0$ for any $n \in \mathbb{N}$.

For the proof we refer to the proof of Theorem 3.7 of [12]. This is a proof by induction on $n$ using $H^{1-r, r+1}\left(\delta_{0}\right)=0$ and $H^{1-r, r+2}\left(\delta_{0}\right)=0$ for $r \geq 2$. Notice that $\delta_{r}=0$ if $r>m^{\prime}$ or $r>m+1$.

THEOREM 4. Let $\delta_{r}(r \geq 0)$ be as above then $\delta=\sum_{r>0} \delta_{r}$ is a differential of the graded $\mathbb{R}$-algebra $\mathcal{K}$ and the cohomology $H(\delta)$ of the graded differential algebra $(\mathcal{K}, \delta)$ identifies with the longitudinal cohomology $H(V, \mathcal{F})$.

Again we refer to 12 (the proof of Theorem 3.8 there); the first part of the statement is obvious, the identification of $H(\delta)$ with $H(V, \mathcal{F})$ follows essentially from an elementary spectral sequence argument.

Let $(M, \omega)$ be a symplectic manifold (i.e. a smooth manifold $M$ equipped with a closed nondegenerate 2-form $\omega$ ) and let $V$ be a closed submanifold of $M$. We denote by $\omega_{V}$ the closed 2-form $i^{*}(\omega)$ on $V$ induced by the inclusion $i: V \rightarrow M$. In general $\omega_{V}$ is degenerate; its characteristic distribution $F$ is the set of tangent vectors $X$ of $V$ such that $i_{X} \omega=0$. It follows from the equation $d \omega_{V}=0$ that the $C^{\infty}(V)$-module $\mathcal{F}$ of vector fields on $V$ which are valued in $F$ is a Lie subalgebra of the Lie algebra of vector fields. Therefore if $\omega_{V}$ is of constant rank, which will be assumed in the sequel, $\mathcal{F}$ is a foliation of $V$. In fact we shall assume not only that $\omega_{V}$ is of constant rank but also that the quotient $V / \mathcal{F}=M_{0}$ is a smooth manifold and that the canonical projection $p: V \rightarrow M_{0}$ is a submersion. With these regularity assumptions, $\omega_{V}$ has a projection $\omega_{0}$ on $M_{0}$ which is, by construction, a closed nondegenerate 2 -form. Thus $\left(M_{0}, \omega_{0}\right)$ is a symplectic manifold which is referred to as the reduced phase space and which is the natural phase space for a hamiltonian 
system on $M$ which is constrained to move on $V$. One has $i^{*}(\omega)=\omega_{V}=p^{*}\left(\omega_{0}\right)$. The algebra of observables of such a constrained system is $C^{\infty}\left(M_{0}\right)$ which identifies with the longitudinal cohomology of degree $0, C^{\infty}\left(M_{0}\right)=H^{0}(V, \mathcal{F})$. Thus if $(M, \omega)$ and $V$ are such that $\left(R_{0}\right)$ is satisfied for $(M, V)$ and $\left(R_{1}\right)$ is satisfied for $(V, \mathcal{F})$, one can use Theorem 4 to compute $C^{\infty}\left(M_{0}\right)$ and more generally $H(V, \mathcal{F})$. The graded differential algebra $\mathcal{K}$ is the ghost complex appropriate to the situation and $\delta$ is the corresponding BRS differential.

The specificity of the above situation is that $\mathcal{F}$ does only depend on the submanifold $V$ of the symplectic manifold $(M, \omega)$; in particular if assumptions $\left(R_{0}\right)$ and $\left(R_{1}\right)$ are satisfied, one can show easily that $m \geq m^{\prime}$ and, on the other hand $m+m^{\prime}=\operatorname{dim}(M)-\operatorname{dim}\left(M_{0}\right)$ is necessarily even since $M$ and $M_{0}$ are both symplectic (and finite-dimensional). The case where the ideal $I(V)$ of smooth functions on $M$ which vanish on $V$ is stable by the Poisson bracket (associated to $\omega$ ) is referred to as the case of first class constraints or the coisotropic case. In such a case, one has $m=m^{\prime}$ and Assumption $\left(R_{0}\right)$ implies $\left(R_{1}\right)$; indeed in this case with Assumption $\left(R_{0}\right)$ the hamiltonian vector fields $\operatorname{Ham}\left(u_{\alpha}\right)$ of the $u_{\alpha}$ have restrictions to $V$ which are tangent to $V$ and form a basis of the $C^{\infty}(V)$-module $\mathcal{F}$, (see e.g. in [12]). This case has the further property that one can extend the Poisson bracket in a superbracket on $\mathcal{K}$ by setting $\left\{\pi_{\alpha}, \chi^{\beta}\right\}=\delta_{\alpha}^{\beta},\left\{\chi^{\alpha}, \chi^{\beta}\right\}=\left\{\pi_{\alpha}, \pi_{\beta}\right\}=0$, $\left\{\pi_{\alpha}, f\right\}=\left\{\chi^{\alpha}, f\right\}=0$ for $f \in C^{\infty}(M)$ and that $\mathcal{K}$ can then be interpreted as the algebra of "functions" on a "super phase space". Moreover in this case the BRS differential $\delta$ can be realized as superhamiltonian, i.e. $\delta \varphi=\{Q, \varphi\}, \forall \varphi \in \mathcal{K}$, for some $Q \in \mathcal{K}$ of total degree 1, [37]. In this case it has been shown in 37] that the arbitrariness of the whole construction is a canonical transformation of the super phase space.

In gauge theory, the usual ghost complex without antighosts was understood early as a Lie algebra cochain complex (see e.g. in [4], [58, [21], [11]) or as a complex of longitudinal forms (see in [64], [59]). This led through the Koszul formula 45. to the interpretation of the corresponding ghosts as components of the MaurerCartan form of the gauge group (see also in $[\mathbf{6 3}$ ). The key of the understanding of the antighost or conjugate ghost part in terms of usual mathematical concepts appears in [48] where it was shown that they provide Koszul resolutions. This led to the homological approach to constrained systems developed e.g. in [52], [56], [12 and [44] in terms of standard mathematical objects which is partly described above.

Assumption $\left(R_{0}\right)$ means regular submanifold $V$. One can generalize the above constructions in several directions without such a regularity. In the case of the first class constrained hamiltonian systems this has been investigated in $\mathbf{5 6}$ and in $\mathbf{2 7}$ where BRS cohomology with ghosts of ghosts has been applied. Finally, it is worth noticing here that an "infinite dimensional" form of Theorem 4 applies directly to the antifield formalism [26], [38] and is also implicit behind the ghost lagrangian formalism of gauge theory [25], [2].

\section{N-complex versions of BRS methods}

The canonical approach to the quantum Wess-Zumino-Novikov-Witten (WZNW) model gives rise to a finite-dimensional quantum group gauge problem 
for the zero modes. This has been studied in a convenient form for us in 31, 32. The result is a finite-dimensional gauge model in which the physical state space appears as a quotient $\mathcal{H}^{\prime} / \mathcal{H}^{\prime \prime}$ where $\mathcal{H}^{\prime}$ is a subspace of the original finitedimensional indefinite metric space whereas $\mathcal{H}^{\prime \prime}$ is the subspace of "null vectors" (isotropic subspace) of $\mathcal{H}^{\prime}$. Using the results of [31], 32], it was shown in 22] that, in the case of the $S U(2)$ WZNW model, the physical state space can be realized as a direct sum $\oplus_{n=1}^{N-1} H_{(n)}\left(\mathcal{H}_{I}, A\right)$ where $\left(H_{(n)}\left(\mathcal{H}_{I}, A\right)\right)$ is the generalized homology of an $N$-differential vector space $\mathcal{H}_{I}$ with $N$-differential $A$. In fact, for the level $k$ representation of the $\widehat{\mathfrak{s u}}(2)$ Kac-Moody algebra, $A$ satisfies $A^{N}=0$ with $N=k+2$. The $N^{4}$-dimensional space $\mathcal{F} \otimes \overline{\mathcal{F}}=\mathcal{H}$ of chiral zero modes carries a representation of the quantum group $U_{q}\left(\mathfrak{s l}_{2}\right) \otimes U_{q}\left(\mathfrak{s l}_{2}\right)$ where $q=e^{i(\pi / N)}$; it is a representation of the usual finite-dimensional quotient $\mathcal{U}_{q}$ of $U_{q}\left(\mathfrak{s l}_{2}\right) \otimes U_{q}\left(\mathfrak{s l}_{2}\right)$ at the primitive root of unity $q\left(q^{2 N}=1\right)$. The $N$-differential $A$ of $\mathcal{H}$ commutes with the action of the Hopf algebra $\mathcal{U}_{q}$ so the $(2 N-1)$-dimensional subspace $\mathcal{H}_{I}$ of $\mathcal{U}_{q}$-invariant vectors is stable by $A$ and it is the generalized homology of the $N$-differential vector space $\left(\mathcal{H}_{I}, A\right)$ which is of interest. In [23] we produced an $N$-differential vector space which contains $\mathcal{H}$ and has the same generalized homology as $\left(\mathcal{H}_{I}, A\right)$. It is this construction which will be explained in a very general setting in what follows.

In short, one has a vector space $\mathcal{H}$ on which act a Hopf algebra $\mathcal{U}_{q}$ and a nilpotent endomorphism $A$ satisfying $A^{N}=0$. The action of the algebra $\mathcal{U}_{q}$ commutes with $A$, i.e. one has on $\mathcal{H}:[A, X]=0, \forall X \in \mathcal{U}_{q}$. It follows that the subspace $\mathcal{H}_{I}$ of $\mathcal{U}_{q}$-invariant vectors in $\mathcal{H}$ is stable by $A$, i.e. $A\left(\mathcal{H}_{I}\right) \subset \mathcal{H}_{I}$. Thus $\left(\mathcal{H}_{I}, A\right)$ is an $N$-differential subspace of the $N$-differential vector space $(\mathcal{H}, A)$ and it turns out that the "interesting object" (the physical space) is the generalized homology of $\left(\mathcal{H}_{I}, A\right)$. We would like to avoid the restriction to the invariant subspace $\mathcal{H}_{I}$ that is, in complete analogy with the BRS methods, we would like to define an extended $N$-differential space in such a way that the $\mathcal{U}_{q}$-invariance is captured by its $N$-differential in the sense that it has the same generalized homology as $\left(\mathcal{H}_{I}, A\right)$. The most natural thing to do is to try to construct a nilpotent endomorphism $Q$ of $\mathcal{H}$ with $Q^{N}=0$ such that its generalized homology coincides with the one of $A$ on $\mathcal{H}_{I}$ i.e. such that one has $H_{(n)}(\mathcal{H}, Q)=H_{(n)}\left(\mathcal{H}_{I}, A\right), \forall n \in\{1, \ldots, N-1\}$. It turns out that this is impossible in general. Indeed in the above case (for the $S U(2)$ WZNW model) $\mathcal{H}$ is finite dimensional and then Proposition 4 (see in Section 5) imposes strong constraints connecting $\operatorname{dim} \mathcal{H}$ and the $\operatorname{dim} H_{(n)}(\mathcal{H}, Q)=\operatorname{dim} H_{(n)}\left(\mathcal{H}_{I}, A\right)$ for $n \in\{1, \cdots, N-1\}$ which are not satisfied [23]. This is not astonishing since in the usual BRS methods one has to add the ghost sector (see e.g. in last section or in Section 4).

We first present an abstract optimal construction in which the Hopf algebra $\mathcal{U}_{q}$ plays no role. We assume that $(\mathcal{H}, A)$ is an $N$-differential vector space, that there is a subspace $\mathcal{H}_{I}$ of $\mathcal{H}$ stable by $A$ and we shall construct an $N$-differential vector space $\left(\mathcal{H}^{\bullet}, Q\right)$ with $\mathcal{H} \subset \mathcal{H}^{\bullet}$ such that $H_{(n)}\left(\mathcal{H}^{\bullet}, Q\right)=H_{(n)}\left(\mathcal{H}_{I}, A\right)$ for all $n \in\{1, \cdots, N-1\}$. Throughout the following $q^{2}$ is still a primitive $N$-th root of unity $\left(q^{N}=-1\right)$. Let us define the graded vector space $\mathcal{H}^{\bullet}=\underset{n \geq 0}{\oplus} \mathcal{H}^{n}$ by $\mathcal{H}^{0}=\mathcal{H}$, $\mathcal{H}^{n}=\mathcal{H} / \mathcal{H}_{I}$ for $1 \leq n \leq N-1$ and $\mathcal{H}^{n}=0$ for $n \geq N$. One then defines an endomorphism $d$ of degree 1 of $\mathcal{H}^{\bullet}$ by setting $d=\pi: \mathcal{H}^{0} \rightarrow \mathcal{H}^{1}$ where $\pi: \mathcal{H} \rightarrow$ $\mathcal{H} / \mathcal{H}_{I}$ is the canonical projection, $d=\operatorname{Id}: \mathcal{H}^{n} \rightarrow \mathcal{H}^{n+1}$ for $1 \leq n \leq N-2$ where Id 
is the identity mapping of $\mathcal{H} / \mathcal{H}_{I}$ onto itself and $d=0$ on $\mathcal{H}^{n}$ for $n \geq N-1$. One has $d^{N}=0$ and therefore $\left(\mathcal{H}^{\bullet}, d\right)$ is an $N$-complex, so its generalized (co)homology is graded $H_{(k)}\left(\mathcal{H}^{\bullet}, d\right)=\underset{n \geq 0}{\oplus} H_{(k)}^{n}\left(\mathcal{H}^{\bullet}, d\right)$. It is given by the following easy lemma.

LEMMA 12. One has $H_{(k)}^{n}\left(\mathcal{H}^{\bullet}, d\right)=0$ for $n \geq 1$ and $H_{(k)}^{0}\left(\mathcal{H}^{\bullet}, d\right)=\mathcal{H}_{I}$, $\forall k \in\{1, \ldots, N-1\}$.

It is worth noticing here that given the vector space $\mathcal{H}$ together with the subspace $\mathcal{H}_{I}$, the $N$-complex $\left(\mathcal{H}^{\bullet}, d\right)$ is characterized (uniquely up to an isomorphism) by the following universal property (the proof of which is straightforward).

LEMMA 13. Any linear mapping $\alpha: \mathcal{H} \rightarrow \mathcal{C}^{0}$ of $\mathcal{H}$ into the subspace $\mathcal{C}^{0}$ of elements of degree 0 of an $N$-complex $\left(\mathcal{C}^{\bullet}, d\right)$ which satisfies $d \circ \alpha\left(\mathcal{H}_{I}\right)=0$ extends uniquely as a homomorphism $\bar{\alpha}:\left(\mathcal{H}^{\bullet}, d\right) \rightarrow\left(\mathcal{C}^{\bullet}, d\right)$ of $N$-complexes.

By using this universal property one can extend $A$ to $\mathcal{H}^{\bullet}$ in the following manner.

LEMMA 14. The endomorphism $A$ of $\mathcal{H}=\mathcal{H}^{0}$ has a unique extension to $\mathcal{H}^{\bullet}$, again denoted by $A$, as a homogeneous endomorphism of degree 0 satisfying $A d-q^{2} d A=0$. On $\mathcal{H}^{\bullet}$, one has $A^{N}=0$ and $(d+A)^{N}=0$.

Thus $Q=d+A$ is an $N$-differential on $\mathcal{H}^{\bullet}$ and we have the following result.

THEOREM 5. The generalized $Q$-homology of $\mathcal{H}^{\bullet}$ coincides with the generalized A-homology of $\mathcal{H}_{I}$, i.e. one has $H_{(k)}\left(\mathcal{H}^{\bullet}, Q\right)=H_{(k)}\left(\mathcal{H}_{I}, A\right)$ for $1 \leq k \leq N-1$.

Notice that $\left(\mathcal{H}^{\bullet}, Q\right)$ is only an $N$-differential vector space and not an $N$-complex since $d+A=Q$ is inhomogeneous.

In the problem of the zero modes of the $S U(2)$ WZNW model, $\mathcal{H}_{I}$ is the invariant subspace of $\mathcal{H}$ by the action of the quantum group (i.e. the Hopf algebra) $\mathcal{U}_{q}$ which plays the role of a gauge group, or more precisely of the universal enveloping algebra of the Lie algebra of a gauge group, so (in view of Theorem 1) it is natural to produce a construction where $\mathcal{U}_{q}$ and its (Hochschild) cohomology enter as in the usual BRS construction for gauge theory in order to get a similar "geometricophysical" interpretation. This is the aim of the end of this section. Since the above construction based on universal property is quite minimal, one cannot be astonished that it occurs as an $N$-differential subspace of the following one.

By definition $\mathcal{H}_{I}$ is the set of $\Psi \in \mathcal{H}$ such that $X \Psi=\Psi \varepsilon(X)$ for any $X \in \mathcal{U}_{q}$, where $\varepsilon$ denotes the counit of $\mathcal{U}_{q}$. This means that if one considers $\mathcal{H}$ as a $\left(\mathcal{U}_{q}, \mathcal{U}_{q}\right)$-bimodule by equipping it with the trivial right action given by the counit, $\mathcal{H}_{I}$ identifies with the $\mathcal{H}$-valued Hochschild cohomology in degree 0 of $\mathcal{U}_{q}$, i.e. $\mathcal{H}_{I}=H^{0}\left(\mathcal{U}_{q}, \mathcal{H}\right)$. The idea of the construction is to mix the Hochschild differential with $A$ in a similar way as the mixing of $\delta_{0}$ with $d_{\mathcal{F}}$ in last section. However, $A$ is an $N$-differential whereas the Hochschild differential is an ordinary differential i.e. a 2-differential. Fortunately the next lemma shows that for the description of $\mathcal{H}_{I}$ one can replace the Hochschild differential by the $N$-differential $d_{1}$ of Section 6 with the replacement of $q$ by $q^{2}$ since here it is $q^{2}$ which is a primitive $N$-th root of unity. To simplify the notations, this $N$-differential $d_{1}$ on $C\left(\mathcal{U}_{q}, \mathcal{H}\right)$ will be denoted 
by $d$. That is the $N$-differential $d$ is defined by

$$
\begin{aligned}
d(\omega)\left(X_{0}, \ldots, X_{n}\right) & =X_{0} \omega\left(X_{1}, \ldots, X_{n}\right) \\
& +\sum_{k=1}^{n} q^{2 k} \omega\left(X_{0}, \ldots,\left(X_{k-1} X_{k}\right), \ldots, X_{n}\right) \\
& -q^{2 n} \omega\left(X_{0}, \ldots, X_{n-1}\right) \varepsilon\left(X_{n}\right) .
\end{aligned}
$$

for $\omega \in C^{n}\left(\mathcal{U}_{q}, \mathcal{H}\right), \quad X_{i} \in \mathcal{U}_{q}$. One has the following lemma.

LEMMA 15. Let $\Psi \in \mathcal{H}=C^{0}\left(\mathcal{U}_{q}, \mathcal{H}\right)$; the following conditions $(i)$, (ii) and (iii) are equivalent

(i) $d^{k}(\Psi)=0$ for some $k$ with $1 \leq k \leq N-1$

(ii) $\Psi \in \mathcal{H}_{I}$

(iii) $d^{n}(\Psi)=0$ for any $n \in\{1, \ldots, N-1\}$.

Observe first that $d\left(=d_{1}\right)$ coincides in degree 0 with the Hochschild differential. Then the result is a consequence of the following formula which one proves by induction on $n$.

$$
d^{n} \Psi(\mathbb{1}, \ldots, \mathbb{1}, X)=\left(1+q^{2}\right) \ldots\left(1+q^{2}+\cdots+q^{2(n-1)}\right) d \Psi(X)
$$

for $\Psi \in C^{0}\left(\mathcal{U}_{q}, \mathcal{H}\right)$ and for any $n \geq 1, X \in \mathcal{U}_{q}$ where $\mathbb{1}$ is the unit of $\mathcal{U}_{q}$.

This lemma implies : $H_{(k)}^{0}\left(C\left(\mathcal{U}_{q}, \mathcal{H}\right), d\right)=H^{0}\left(\mathcal{U}_{q}, \mathcal{H}\right), \forall k \in\{1, \ldots, N-1\}$. This is a special case of Theorem 2 of Section 6. As an easy consequence, one obtains the following result.

PROPOSITION 8. The $N$-complex $\left(\mathcal{H}^{\bullet}, d\right)$ can be canonically identified with the $N$-subcomplex of $\left(C\left(\mathcal{U}_{q}, \mathcal{H}\right), d\right)$ generated by $\mathcal{H}$.

Thus one has $\mathcal{H}^{\bullet} \subset C\left(\mathcal{U}_{q}, \mathcal{H}\right)$ and the $N$-differential $d$ of $C\left(\mathcal{U}_{q}, \mathcal{H}\right)$ extends the one of $\mathcal{H}^{\bullet}$; we now extend $A$ to $C\left(\mathcal{U}_{q}, \mathcal{H}\right)$.

LEMMA 16. Let us extend $A$ to $C\left(\mathcal{U}_{q}, \mathcal{H}\right)$ as a homogeneous endomorphism $\omega \mapsto(A \omega)$ of degree 0 by setting

$$
(A \omega)\left(X_{1}, \ldots, X_{n}\right)=q^{2 n} A \omega\left(X_{1}, \ldots, X_{n}\right)
$$

for $\omega \in C^{n}\left(\mathcal{U}_{q}, \mathcal{H}\right)$ and $X_{i} \in \mathcal{U}_{q}$. On $C\left(\mathcal{U}_{q}, \mathcal{H}\right)$ one has $A d-q^{2} d A=0, A^{N}=0$ and $(d+A)^{N}=0$.

We have now extended to $C\left(\mathcal{U}_{q}, \mathcal{H}\right)$ the whole structure defined previously on $\mathcal{H}^{\bullet}$. Indeed the uniqueness in Lemma 14 implies that $A$ defined on $C\left(\mathcal{U}_{q}, \mathcal{H}\right)$ in last lemma is an extension of $A$ defined on $\mathcal{H}^{\bullet}$ in Lemma 14 . One then extends to $C\left(\mathcal{U}_{q}, \mathcal{H}\right)$ the definition of $Q$ by setting again $Q=d+A$.

As explained in Section 6, Theorem $2(1)$, the spaces $H_{(k)}^{n}\left(C\left(\mathcal{U}_{q}, \mathcal{H}\right), d\right)$ can be computed in terms of the Hochschild cohomology $H\left(\mathcal{U}_{q}, \mathcal{H}\right)$. In particular, one sees that $H_{(k)}^{n}\left(C\left(\mathcal{U}_{q}, \mathcal{H}\right), d\right)$ does not generally vanish for $n \geq 1$. This implies that one cannot expect for the generalized homology of $Q$ on $C\left(\mathcal{U}_{q}, \mathcal{H}\right)$ such a simple result as the one given by Theorem 5 for the generalized homology of $Q$ on $\mathcal{H}^{\bullet}$. Nevertheless, in view of Lemma 15, one has $H_{(k)}^{0}\left(C\left(\mathcal{U}_{q}, \mathcal{H}\right), d\right)=\mathcal{H}_{I}=H_{(k)}^{0}\left(\mathcal{H}^{\bullet}, d\right)$ and therefore one may expect $H_{(k)}^{0}\left(C\left(\mathcal{U}_{q}, \mathcal{H}\right), Q\right)=H_{(k)}\left(\mathcal{H}_{I}, A\right)\left(=H_{(k)}\left(\mathcal{H}^{\bullet}, Q\right)\right)$. In fact, this is essentially true. However some care must be taken because $Q$ is not homogeneous so $H_{(k)}\left(C\left(\mathcal{U}_{q}, \mathcal{H}\right), Q\right)$ is not a graded vector space. Instead of a 
graduation, one has an increasing filtration $F^{n} H_{(k)}\left(C\left(\mathcal{U}_{q}, \mathcal{H}\right), Q\right),(n \in \mathbb{Z})$, with $F^{n} H_{(k)}\left(C\left(\mathcal{U}_{q}, \mathcal{H}\right), Q\right)=0$ for $n<0$ and where, for $n \geq 0, F^{n} H_{(k)}\left(C\left(\mathcal{U}_{q}, \mathcal{H}\right), Q\right)$ is the canonical image in $H_{(k)}\left(C\left(\mathcal{U}_{q}, \mathcal{H}\right), Q\right)$ of $\operatorname{Ker}\left(Q^{k}\right) \cap \underset{r=0}{r=n} C^{r}\left(\mathcal{U}_{q}, \mathcal{H}\right)$. There is an associated graded vector space

$$
{ }^{g r} H_{(k)}\left(C\left(\mathcal{U}_{q}, \mathcal{H}\right), Q\right)=\oplus_{n} F^{n} H_{(k)}\left(C\left(\mathcal{U}_{q}, \mathcal{H}\right), Q\right) / F^{n-1} H_{(k)}\left(C\left(\mathcal{U}_{q}, \mathcal{H}\right), Q\right)
$$

which here is $\mathbb{N}$-graded. One has $F^{0} H_{(k)}\left(C\left(\mathcal{U}_{q}, \mathcal{H}\right), Q\right)={ }^{\mathrm{g} r} H_{(k)}^{0}\left(C\left(\mathcal{U}_{q}, \mathcal{H}\right), Q\right)$ and it is this space which is the correct version of the $H_{(k)}^{0}\left(C\left(\mathcal{U}_{q}, \mathcal{H}\right), Q\right)$ above in order to identify $H_{(k)}\left(\mathcal{H}_{I}, A\right)$ in the generalized homology of $Q$ on $C\left(\mathcal{U}_{q}, \mathcal{H}\right)$.

THEOREM 6. The inclusion $\mathcal{H}^{\bullet} \subset C\left(\mathcal{U}_{q}, \mathcal{H}\right)$ induces the isomorphisms

$$
H_{(k)}\left(\mathcal{H}^{\bullet}, Q\right) \simeq F^{0} H_{(k)}\left(C\left(\mathcal{U}_{q}, \mathcal{H}\right), Q\right) \text { for } 1 \leq k \leq N-1 .
$$

In particular, with obvious identifications, one has

$$
F^{0} H_{(k)}\left(C\left(\mathcal{U}_{q}, \mathcal{H}\right), Q\right)=H_{(k)}\left(\mathcal{H}_{I}, A\right), \quad \forall k \in\{1, \ldots, N-1\} .
$$

The proof is not difficult, for it as well as for complete proofs of all the results of this section we refer to 23 .

If one compares this construction involving Hochschild cochains with the preceeding one, what has been gained here besides the explicit occurrence of the quantum gauge aspect is that the extended space $C\left(\mathcal{U}_{q}, \mathcal{H}\right)$ is a tensor product $\mathcal{H} \otimes \mathcal{H}^{\prime}$ of the original space $\mathcal{H}$ with the tensor algebra $\mathcal{H}^{\prime}=T\left(\mathcal{U}_{q}^{*}\right)$ of the dual space of $\mathcal{U}_{q}$. The factor $\mathcal{H}^{\prime}$ can thus be interpreted as the state space for some generalized ghost. What has been lost is the minimality of the generalized homology, i.e. besides the "physical" $H_{(k)}\left(\mathcal{H}_{I}, A\right)$, the generalized homology of $Q$ on $\mathcal{H} \otimes \mathcal{H}^{\prime}$ contains some other non trivial subspace in contrast to what happens on $\mathcal{H}^{\bullet}$. In the usual homological (BRS) methods however such a "non minimality" also occurs. Indeed,as explained in last section, in the homological approach to constrained classical systems, the relevant homology contains besides the functions on the reduced phase space the whole cohomology of longitudinal forms. The same is true for the BRS cohomology of gauge theory [2], [4].

In the usual situations where one applies the BRS construction (gauge theory, constrained systems) one has a Lie algebra $\mathfrak{g}$ (the Lie algebra of infinitesimal gauge transformations) acting on some space $\mathcal{H}$ and what is really relevant at this stage is the Lie algebra cohomology $H(\mathfrak{g}, \mathcal{H})$ of $\mathfrak{g}$ acting on $\mathcal{H}$. The extended space is then the space of $\mathcal{H}$-valued Lie algebra cochains of $\mathfrak{g}, C(\mathfrak{g}, \mathcal{H})$. This extended space is thus also a tensor product $\mathcal{H} \otimes \mathcal{H}^{\prime}$ but now $\mathcal{H}^{\prime}$ is the exterior algebra $\mathcal{H}^{\prime}=\Lambda \mathfrak{g}^{*}$ of the dual space of $\mathfrak{g}$. That is why this factor can be interpreted (due to antisymmetry) as a fermionic state space; indeed that is the reason why one gives a fermionic character to the ghost [2], [4]. There is however another way to proceed in these situations which is closer to what has been done in our case here. To understand it, we recall that any representation of $\mathfrak{g}$ in $\mathcal{H}$ is also a representation of the enveloping algebra $U(\mathfrak{g})$ in $\mathcal{H}$. Thus $\mathcal{H}$ is a left $U(\mathfrak{g})$-module. Since $U(\mathfrak{g})$ is a Hopf algebra, one can convert as above $\mathcal{H}$ into a bimodule for $U(\mathfrak{g})$ by taking as right action the trivial representation given by the counit. It turns out that as explained in Section 3, Theorem 1, the $\mathcal{H}$-valued Hochschild cohomology of $U(\mathfrak{g}), H(U(\mathfrak{g}), \mathcal{H})$, coincides with the $\mathcal{H}$-valued Lie algebra cohomology of $\mathfrak{g}, H(\mathfrak{g}, \mathcal{H})$. Since it is the latter space which is relevant one can as well take as extended space the space of 
$\mathcal{H}$-valued Hochschild cochains of $U(\mathfrak{g}), C(U(\mathfrak{g}), \mathcal{H})$, and then compute its cohomology. Again this space is a tensor product $\mathcal{H} \otimes \mathcal{H}^{\prime}$ but now $\mathcal{H}^{\prime}=T\left(U(\mathfrak{g})^{*}\right)$ is a tensor algebra as in our case.

\section{Acknowledgments}

I thank Robert Coquereaux and Raymond Stora for their constructive critical reading of the manuscript.

\section{Appendix A. Remarks on tensor products}

Let $\wedge\{d\}$ be the associative unital k-algebra generated by an element $d$ satisfying $d^{2}=0$. As a $\mathbf{k}$-algebra $\wedge\{d\}=\mathbf{k} \mathbb{1} \oplus \mathbf{k} d$ is the exterior algebra (over $\mathbf{k}$ ) of the free $\mathbf{k}$-module of rank one. It is clear that a $\wedge\{d\}$-module is the same thing as a differential module (as defined in Section 2). Given two differential modules $E$ and $F$ there is a canonical structure of $\wedge\{d\} \otimes \wedge\{d\}$-module on $E \otimes F$, where the first factor (resp. the second factor) corresponds to the structure of $\wedge\{d\}$-module of $E$ (resp. of $F$ ). To say that for any such $E$ and $F$ there is a canonical differential on $E \otimes F$ (i.e. a canonical structure of $\wedge\{d\}$-module on $E \otimes F$ ) which only depends on the differentials of $E$ and $F$ (i.e. on their $\wedge\{d\}$-module structures) is the same thing as to say that one has a coproduct $\Delta$ on $\wedge\{d\}$, that is a homomorphism of unital k-algebras $\Delta: \wedge\{d\} \rightarrow \wedge\{d\} \otimes \wedge\{d\}$. One must have $\Delta(\mathbb{1})=\mathbb{1} \otimes \mathbb{1}$ so $\Delta$ is fixed by giving a $\Delta(d) \in \wedge\{d\} \otimes \wedge\{d\}$ satisfying $(\Delta(d))^{2}=0$. One has $\Delta(d)=\alpha \mathbb{1} \otimes \mathbb{1}+\beta \mathbb{1} \otimes d+\gamma d \otimes \mathbb{1}+\delta d \otimes d$ with $\alpha, \beta, \gamma, \delta \in \mathbf{k}$ and $(\Delta(d))^{2}=0$ implies $\alpha^{2}=0,2 \alpha \beta=0,2 \alpha \gamma=0$ and $2(\alpha \delta+\beta \gamma)=0$.

Let us now assume that $\mathbf{k}$ is a field of characteristic different of 2 . Then the above conditions imply $\alpha=0$ and $\beta \gamma=0$ i.e. either $\Delta(d)=\beta \mathbb{1} \otimes d+\delta d \otimes d$ or $\Delta(d)=\gamma d \otimes \mathbb{1}+\delta d \otimes d$. It is already clear that generically the differential $\beta \mathbb{1} \otimes d+\delta d \otimes d($ resp. $\gamma d \otimes \mathbb{1}+\delta d \otimes d)$ on $E \otimes F$ will lead to a homology $H(E \otimes F)$ for $E \otimes F$ different of $H(E) \otimes H(F)$. Notice that if one imposes the natural requirement of coassociativity for $\Delta$ one is led to the only 3 possibilities $\mathbb{1} \otimes d, d \otimes \mathbb{1}$ or $d \otimes d$ for the differential on the tensor products.

Let us come back to a general ring $\mathbf{k}$. Consider the associative unital $\mathbf{k}$-algebra $\mathcal{D}_{-1}$ generated by two elements $d$ and $\Gamma$ satisfying $d^{2}=0, \Gamma^{2}=\mathbb{1}$ and $\Gamma d=-d \Gamma$. This algebra is a Hopf algebra for the counit $\varepsilon$, the antipode $S$ and the coproduct $\Delta$ given by : $\varepsilon(d)=0, \varepsilon(\Gamma)=1, S(d)=-\Gamma d, S(\Gamma)=\Gamma, \Delta(d)=d \otimes \mathbb{1}+\Gamma \otimes d$ and $\Delta(\Gamma)=\Gamma \otimes \Gamma$. The Hopf algebra $\mathcal{D}_{-1}$ can be understood as a version of the universal enveloping algebra of the super Lie algebra with only one odd element $d$ such that $[d, d]=0$. Let $E=E^{0} \oplus E^{1}$ be a $\mathbb{Z}_{2}$-complex then $E$ is a $\mathcal{D}_{-1}$-module if $d$ is represented by the differential of $E$ and if $\Gamma$ is represented by the multiplication by $(-1)^{i}$ on $E^{i}$ for $i \in\{0,1\}$. One verifies easily that the tensor product of $\mathbb{Z}_{2}$-complexes defined in Section 3 corresponds to the above structure, i.e. that it is induced by the coproduct $\Delta$. Thus one can understand the tensor product of complexes in terms of a Hopf algebra. We now show that the same is true for $N$-complexes.

Let $q \in \mathbf{k}$ be such that Assumption $\left(A_{1}\right)$ of Section 5 is satisfied and let us consider the associative unital $\mathbf{k}$-algebra $\mathcal{D}_{q}$ generated by two elements $d$ and $\Gamma$ 
satisfying $d^{N}=0, \Gamma^{N}=\mathbb{1}$ and $\Gamma d=q d \Gamma$. Again $\mathcal{D}_{q}$ is a Hopf algebra for the counit $\varepsilon$, the antipode $S$ and the coproduct $\Delta$ given by : $\varepsilon(d)=0, \varepsilon(\Gamma)=1$, $S(d)=-\Gamma^{N-1} d, S(\Gamma)=\Gamma^{N-1}, \Delta(d)=d \otimes \mathbb{1}+\Gamma \otimes d$ and $\Delta \Gamma=\Gamma \otimes \Gamma$. Let $E=E^{0} \oplus \cdots \oplus E^{N-1}$ be a $\mathbb{Z}_{N}$-complex (see in Section 6) then $E$ is a $\mathcal{D}_{q}$-module if $d$ is represented by the $N$-differential of $E$ and $\Gamma$ is represented by the multiplication by $q^{i}$ on $E^{i}$ for $i \in\{0, \ldots, N-1\}$. Again one verifies easily that the $(q)$

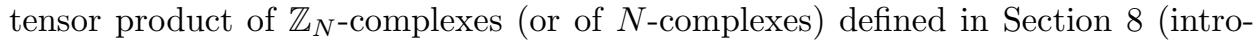
duced originally in [40]) is induced by the coproduct of $\mathcal{D}_{q}$.

Finally it is worth noticing here that instead of $\mathcal{D}_{-1}$ one can use the exterior algebra of the free module of rank one $\wedge^{\bullet}\{d\}=\wedge \mathbf{k}=\mathbf{k} \mathbb{1} \oplus \mathbf{k} d$ considered as a graded Hopf algebra. That is, as an associative algebra $\wedge \bullet\{d\}$ is isomorphic to $\wedge\{d\}$ but it is a $\mathbb{Z}_{2}$-graded algebra with $\wedge^{0}\{d\}=\mathbf{k} \mathbb{1}, \wedge^{1}\{d\}=\mathbf{k} d$ and furthermore it is a graded Hopf algebra for the counit $\varepsilon$, the antipode $S$ and the coproduct $\Delta$ given by $\varepsilon(d)=0, S(d)=-d$ and $\Delta d=d \otimes \mathbb{1}+\mathbb{1} \otimes d$ where now $\Delta$ is a homomorphism of graded algebras of $\wedge^{\bullet}\{d\}$ into $\wedge^{\bullet}\{d\} \otimes \wedge^{\bullet}\{d\}$ with $\wedge^{\bullet}\{d\} \otimes \wedge^{\bullet}\{d\}$ being the (twisted) tensor product of graded algebras defined in Section 8. A $\mathbb{Z}_{2}$-complex is canonically a graded $\wedge \bullet\{d\}$-module and the tensor product of complexes can be also defined by using the above graded coproduct. Of course $\wedge^{\bullet}\{d\}$ is also a version (which is graded) of the universal enveloping algebra of the super Lie algebra with one odd generator $d$ satisfying $[d, d]=0$. The advantage of $\mathcal{D}_{-1}$ is that it generalizes as $\mathcal{D}_{q}$ for $N$-complexes as explained above and that it is an ordinary Hopf algebra (in fact $\Gamma$ plays the role of the graduation).

\section{Appendix B. Longitudinal forms}

Let $\mathcal{A}$ be an associative unital algebra over $\mathbb{R}$ or $\mathbb{C}$ (here $\mathbf{k}=\mathbb{R}$ or $\mathbb{C}$ ) and let us denote by $Z(\mathcal{A})$ the center of $\mathcal{A}$ that is the commutative unital subalgebra of $\mathcal{A}$ defined by $Z(\mathcal{A})=\{z \in \mathcal{A} \mid z a=a z, \quad \forall a \in \mathcal{A}\}$. One has $\mathcal{A}=Z(\mathcal{A})$ if and only if $\mathcal{A}$ is commutative. Recall that a derivation of $\mathcal{A}$ is a linear mapping $X: \mathcal{A} \rightarrow \mathcal{A}$ such that one has (Leibniz rule) $X(a b)=X(a) b+a X(b), \forall a, b \in \mathcal{A}$. If $X$ and $Y$ are derivations of $\mathcal{A}$, their composition $X Y$ (product in $\operatorname{End}(\mathcal{A}))$ is not a derivation but the commutator $[X, Y]=X Y-Y X$ is again a derivation of $\mathcal{A}$. On the other hand, if $X$ is a derivation of $\mathcal{A}$ and if $z$ is in the center of $\mathcal{A}$ then $z X$ (defined by $(z X)(a)=z X(a), \quad \forall a \in \mathcal{A})$ is again a derivation of $\mathcal{A}$. Thus the vector space $\operatorname{Der}(\mathcal{A})$ of all derivations of $\mathcal{A}$ is a Lie algebra (for $[\cdot, \cdot])$ and also a $Z(\mathcal{A})$-module, both structures being connected through the identity

$(D Z) \quad[X, z Y]=z[X, Y]+X(z) Y$

for any derivation $X$ and $Y$ and for any $z \in Z(\mathcal{A})$; one verifies easily that the center is stable by derivation i.e. that one has $X(z) \in Z(\mathcal{A})$ for any $X \in \operatorname{Der}(\mathcal{A})$ and $z \in Z(\mathcal{A})$. Let $\mathcal{F} \subset \operatorname{Der}(\mathcal{A})$ be a $Z(\mathcal{A})$-submodule which is also a Lie subalgebra of $\operatorname{Der}(\mathcal{A})$. The graded space $C_{\wedge}(\mathcal{F}, \mathcal{A})$ of (Chevalley-Eilenberg) $\mathcal{A}$-valued cochains of the Lie algebra $\mathcal{F}$ (see in Section 3 ) is canonically a graded algebra and, since $\mathcal{F}$ operates by derivation on $\mathcal{A}$, the corresponding Chevalley-Eilenberg differential $d$ is an antiderivation of $C_{\wedge}(\mathcal{F}, \mathcal{A})$. Thus $C_{\wedge}(\mathcal{F}, \mathcal{A})$ is a graded differential algebra (see in Section 8). It follows from the above identity $(D Z)$ that the graded subalgebra $\underline{\Omega}_{\mathcal{F}}(\mathcal{A})$ of cochains which are $Z(\mathcal{A})$-multilinear is stable by $d$ so $\underline{\Omega}_{\mathcal{F}}(\mathcal{A})$ is a graded differential algebra. 
Let $V$ be a smooth manifold and let $\mathcal{F}$ be a foliation of $V$ (see in Section 9), then the graded differential algebra $\underline{\Omega}_{\mathcal{F}}\left(C^{\infty}(V)\right)$ is referred to as the graded differential algebra of longitudinal forms and is denoted by $\Omega(V, \mathcal{F})$; its elements are called longitudinal forms. This is the graded differential algebra considered in Section 9. Notice that when $\mathcal{F}$ coincides with the module $\operatorname{Der}\left(C^{\infty}(V)\right)$ of all vector fields on $V$ then $\underline{\Omega}_{\text {Der }}\left(C^{\infty}(V)\right)=\underline{\Omega}_{\operatorname{Der}\left(C^{\infty}(V)\right)}\left(C^{\infty}(V)\right)$ is the graded differential algebra $\Omega(V)$ of differential forms on $V$. This is why $\underline{\Omega}_{\operatorname{Der}}(\mathcal{A})=\underline{\Omega}_{\operatorname{Der}(\mathcal{A})}(\mathcal{A})$ is a noncommutative generalization of the graded differential algebra of differential forms when $\mathcal{A}$ is noncommutative; there are other noncommutative generalizations of differential forms (see e.g. in [16]).

\section{References}

[1] H. Araki, Indecomposable representations with invariant inner product, Commun. Math. Phys.97 (1985) 149-159.

[2] C. Becchi, A. Rouet, R. Stora, Renormalization models with broken symmetries in "Renormalization Theory", Erice 1975, G. Velo, A.S. Wightman Eds, Reidel 1976.

[3] C. Becchi, A. Rouet, R. Stora, Renormalization of gauge theories, Ann. Phys. 98 (1976) 287-321.

[4] L. Bonora, P. Cotta-Ramusino, Some remarks on BRS transformations, anomalies and the cohomology of the Lie algebra of the group of gauge transformations, Commun. Math. Phys. 87 (1983) 589-603.

[5] S. Boukraa, The BRS algebra of a free minimal differential algebra, Nucl. Phys. B303 (1988) 237-259.

[6] H. Cartan, Notion d'algèbre différentielle; application aux groupes de Lie et aux variétés où opère un groupe de Lie and La trangression dans un groupe de Lie et dans un espace fibré principal, Colloque de topologie (Bruxelles 1950), Paris, Masson 1951.

[7] H. Cartan, S. Eilenberg, Homological algebra, Princeton University Press 1973.

[8] R. Coquereaux, Noncommutative geometry and theoretical physics, J. Geom. Phys. 6 (1989) 425-490.

[9] T. Damour, S. Deser, Geometry of spin 3 gauge theories, Ann. Inst. H. Poincaré 47 (1987) 277-307.

[10] B. de Wit, D.Z. Freedman, Systematics of higher-spin gauge fields, Phys. Rev.D21 (1980) 358-367.

[11] M. Dubois-Violette, The Weil-BRS algebra of a Lie algebra and the anomalous terms in gauge theory, J. Geom. Phys., 3 (1987) 525-565.

[12] M. Dubois-Violette, Systèmes dynamiques contraints : l'approche homologique, Ann. Inst. Fourier Grenoble 37 (1987) 45-57.

[13] M. Dubois-Violette, Generalized differential spaces with $d^{N}=0$ and the $q$-differential calculus, Czech J. Phys.46 (1997) 1227-1233.

[14] M. Dubois-Violette, $d^{N}=0$ : Generalized homology, K-Theory 14 (1998) 371-404.

[15] M. Dubois-Violette, Generalized homologies for $d^{N}=0$ and graded $q$-differential algebras, Contemporary Mathematics 219 (1998) 69-79.

[16] M. Dubois-Violette, Lectures on graded differential algebras and noncommutative geometry, math.QA/9912017.

[17] M. Dubois-Violette, M. Henneaux, Generalized cohomology for irreducible tensor fields of mixed Young symmetry type, Lett. Math. Phys. 49 (1999) 245-252.

[18] M. Dubois-Violette, M. Henneaux, Tensor fields of mixed Young symmetry type and Ncomplexes, in preparation.

[19] M. Dubois-Violette, M. Henneaux, M. Talon, C.M. Viallet, General solution of the consistency equation, Phys. Lett. B289 (1992) 361-367.

[20] M. Dubois-Violette, R. Kerner, Universal q-differential calculus and q-analog of homological algebra, Acta Math. Univ. Comenian. 65 (1996) 175-188.

[21] M. Dubois-Violette, M. Talon, C.M. Viallet, B.R.S. algebras. Analysis of consistency equations in gauge theory, Commun. Math. Phys. 102 (1985) 105-122.

[22] M. Dubois-Violette, I.T. Todorov, Generalized cohomology and the physical subspace of the SU(2) WZNW model, Lett. Math. Phys. 42 (1997) 183-192. 
[23] M. Dubois-Violette, I.T. Todorov, Generalized homology for the zero mode of the SU(2) WZNW model, Lett. Math. Phys. 48 (1999) 323-338.

[24] H. Epstein, V. Glaser, The role of locality in perturbation theory, Ann. Inst. Henri Poincaré A19 (1973) 211-295.

[25] L.D. Faddeev, V.N. Popov, Feynman diagrams for the Yang-Mills field, Phys. Lett. 25B (1967) 29-30.

[26] J.M.L. Fisch, M. Henneaux, Homological perturbation theory and the algebraic structure of the antifield-antibracket formalism for gauge theories, Commun. Math. Phys. 128 (1990) 627-640.

[27] J.M.L. Fisch, M. Henneaux, J. Stasheff, C. Teitelboim, Existence, uniqueness and cohomology of the classical BRST charge with ghosts of ghosts, Commun. Math. Phys. 120 (1989) 379407.

[28] E.S. Fradkin, G.A. Vilkovisky, Quantization of relativistic systems with constraints, Phys. Lett. B55 (1975) 224-226.

[29] C. Fronsdal, Massless fields with integer spins, Phys. Rev. D 18 (1978) 3624-3629.

[30] W. Fulton, Young tableaux, Cambridge University Press 1997.

[31] P. Furlan, L.K. Hadjiivanov, I.T. Todorov, Operator realization of the $S U(2) W Z N W$ model, Nucl. Phys. B474 (1996) 497-511.

[32] P. Furlan, L.K. Hadjiivanov, I.T. Todorov, A quantum gauge group approach to the $2 D$ $S U(N)$ WZNW model, Int. J. Mod. Phys. A12 (1997) 23-32.

[33] J. Gasqui, Sur les structures de courbure d'ordre 2 dans $\mathbb{R}^{n}$, J. Differential Geometry 12 (1977) 493-497.

[34] H.S. Green, A generalized method of field quantization, Phys. Rev. 90 (1953) 270-273.

[35] W. Greub, Linear algebra, Springer-Verlag 1963.

[36] W. Greub, S. Halperin, R. Vanstone, Connections, curvature, and cohomology, Vol. III, Academic Press 1976.

[37] M. Henneaux, Hamiltonian form of the path integral for theories with a gauge freedom, Physics Reports 126 (1985) 1-66.

[38] M. Henneaux, C. Teitelboim, Quantization of gauge systems, Princeton University Press 1992.

[39] C. Itzykson, J.B. Zuber, Quantum field theory, McGraw-Hill Inc., 1980.

[40] M.M. Kapranov, On the q-analog of homological algebra, Preprint Cornell University 1991, q-alg/9611005.

[41] M. Karoubi, Homologie cyclique des groupes et algèbres, C.R. Acad. Sci. Paris 297, Série I, (1983) 381-384.

[42] M. Karoubi, Homologie cyclique et K-théorie, Astérisque 149 (SMF), 1987.

[43] C. Kassel, M. Wambst, Algèbre homologique des $N$-complexes et homologies de Hochschild aux racines de l'unité Publ. RIMS, Kyoto Univ. 34 (1998) 91-114.

[44] B. Kostant, S. Sternberg, Symplectic reduction, BRS cohomology and infinite-dimensional Clifford algeras, Ann. Physics 176 (1987) 49-113.

[45] J.L. Koszul, Homologie et cohomologie des algèbres de Lie, Bull. Soc. Math. Fr. 78 (1950) 65-127.

[46] J.P. Labesse, Champs libres: Cas de l'électron de Dirac et du photon. Exposés à Dijon 1980.

[47] J.-L. Loday, Cyclic homology, Springer-Verlag, New York 1992.

[48] D. McMullan, Constraints and B.R.S. symmetry, Imperial College Preprint TP 83-84/21 (1984).

A.D. Browning, D. McMullan, The Batalin-Fradkin-Vilkovsky formalism for higher-order theories, J. Math. Phys. 28 (1987) 438-444.

[49] W. Mayer, A new homology theory I, II, Annals of Math. 43 (1942) 370-380 and 594-605.

[50] N. Nakanishi, I. Ojima, Covariant operator formalism of gauge theories and quantum gravity, World Scientific 1990.

[51] G. Rideau, Noncompletely reductible representations of the Poincaré group associated with the generalized Lorentz gauge, J. Math. Phys. 19 (1978) 1627-1634.

[52] M. Rosso, Espace des phases réduit et cohomologie B.R.S., Adv. Ser. Math. Phys. 4 (1988) 263-269.

[53] B. Schroer, A course on: An alqebraic approach to nonperturbative quantum field theory, CBPF, Rio de Janeiro 1998, hep-th 9707230 revised. 
[54] L.P.S. Singh, C.R. Hagen, Lagrangian formulation for arbitrary spin. 1. The boson case, Phys. Rev. D 9 (1974) 898-909.

[55] A. Sitarz, On the tensor product construction for q-differential algebras, Lett. Math. Phys. 44 (1998) 17-21.

[56] J.D. Stasheff, Constrained hamiltonians: a homological approach, Suppl. Rendiconti del Circ. Mat. di Palermo, Proc. Winter School on Geometry and Physics (1987) 239-252.

[57] J.D. Stasheff, Homological (ghost) methods in mathematical physics in "Infinite dimensional geometry, non commutative geometry, operator algebras, fundamental interactions", Saint François - Guadeloupe 1993, R. Coquereaux, M. Dubois-Violette, P. Flad Eds, World Scientific 1995.

[58] R. Stora, Algebraic structure of chiral anomalies in "New perspectives in quantum field theories", Jaca (Spain) 1985, World Scientific 1986.

[59] R. Stora, De la fixation de jauge considérée comme un des beaux arts et de la symétrie de Slavnov qui s'ensuit, hep-th/9611115.

[60] R. Stora, Exercises in equivariant cohomology in "Quantum fields and quantum space-time", Cargèse 1996, G. 't Hooft, A. Jaffe, G. Mack, P.K. Mitter, R. Stora Eds, Plenum Press 1997.

[61] F. Strocchi, Locality and covariance in qed and gravitation. General proof of Gupta-Bleuler type formulations, Lectures in Theoretical Physics, Vol. XIV B, Boulder, Colorado 1973.

[62] F. Strocchi, A.S. Wightman, Proof of the charge superselection rule in local relativistic quantum field theory, J. Math. Phys. 15 (1974) 2198-2224.

63] D. Sullivan, Infinitesimal computations in topology, Publ. IHES 47 (1977) 269-331.

[64] C.-M. Viallet, The geometry of the space of fields in Yang-Mills theory in "Fields and geometry", Karpacz (Poland) 1986, A. Jadczyk Ed., World Scientific 1986.

[65] R. M. Wald, Spin-two fields and general covariance, Phys. Rev. D 33 (1986) 3613-3625.

[66] C.A. Weibel, An introduction to homological algebra, Cambridge University Press 1994.

[67] S. Weinberg, The quantum theory of fields, I, II, Cambridge University Press 1996.

Laboratoire de Physique ThéoriquA 1 , Bâtiment 210, Université Paris Xi, F-91405 Orsay Cedex

E-mail address: patricia@osiris.th.u-psud.fr

\footnotetext{
${ }^{1}$ Unité Mixte de Recherche du Centre National de la Recherche Scientifique - UMR 8627
} 